\title{
Series expansion of complex ground amplifications with a sequence of simple transfer functions
}

\author{
$\operatorname{AUTHOR}(\mathrm{S}):$ \\ Goto, Hiroyuki
}

\section{CITATION:}

Goto, Hiroyuki. Series expansion of complex ground amplifications with a sequence of simple transfer functions. Earthquake Engineering \& Structural Dynamics 2014, 43(13): 1975-1997

\section{ISSUE DATE:}

\section{4-04-24}

URL:

http://hdl.handle.net/2433/198279

\section{RIGHT:}

This is the peer reviewed version of the following article: Goto H. (2014), Series expansion of complex ground amplifications with a sequence of simple transfer functions, Earthquake Engng Struct. Dyn., 43, pages 1975-1997, which has been published in final form at http://dx.doi.org/10.1002/eqe.2432

This article may be used for non-commercial purposes in accordance with Wiley Terms and Conditions for SelfArchiving.; This is not the published version. Please cite only the published version.; この論文は出版社版でありません 。引用の際には出版社版をご確認じ利用ください。 


\title{
Series expansion of complex ground amplifications with a sequence of simple transfer functions
}

\author{
Hiroyuki Goto* \\ Disaster Prevention Research Institute, Kyoto University, Gokasho, Uji, Kyoto 611-0011, Japan
}

\begin{abstract}
SUMMARY
Amplification of earthquake ground motions at actual deposit sites is an important factor to consider when assessing the risk of an earthquake disaster. In order to identify the amplification properties, several preprocessings such as the Fourier transform are required. I propose a series expansion of the amplification with simple ground transfer functions as a new preprocessing. I define a sequence of transfer functions based on a two-layered structure excluding an internal damping, and a function space spanned by the set of the functions. I mathematically prove that the function space is equal to $L^{2}$ space. This indicates that all the functions belonging to $L^{2}$ space, i.e., an arbitrary ground amplification, have a unique series expansion. This expansion is applied to the physics-based decomposition of the amplification. Some numerical examples indicate that the similarity between a target complex structure and a simple model is measured by the absolute value of each coefficient in the series expansion. Copyright (c) 2014 John Wiley \& Sons, Ltd.
\end{abstract}

Received ...

KEY WORDS: ground transfer function; site amplification; series expansion; wave propagation

\section{INTRODUCTION}

In earthquake engineering, amplification of a seismic wave owing to ground surface layers is one of the important factors contributing to an earthquake disaster [1]. Differences in the amplification lead to a spatial variation in ground motions. This has sometimes caused structural damage to be concentrated in a localized area during historical earthquakes. During the 1995 Kobe earthquake, more than $30 \%$ of the wooden houses collapsed in a particular area in the southern part of Hyogo prefecture. The cause was recognized as the basin-edge effect, which is the interference in the seismic waves induced by the irregular shape of the bedrock [2]. A similar phenomenon was observed in Adapazari during the 1999 Kocaeli, Turkey earthquake. Results of numerical simulation indicate that the 3D basin structure beneath the town caused a large amplification of ground motions [3]. Also, during the 2011 off the Pacific coast of Tohoku earthquake, structural damage due to ground motion occurred locally inland of the Tohoku area [4]. In Furukawa district, the damage was severe. A very dense seismic array is now operating in the area in order to investigate the difference in the ground amplifications, and the reason for the localized damage within the area [5].

In order to assess the risk of an earthquake disaster prior to its occurrence, several organizations recently have attemted to quantify the ground amplification across the earth. The objective is to estimate the hazard risk at a particular region [6], and incorporate it into earthquake disaster

\footnotetext{
${ }^{*}$ Correspondence to: Hiroyuki Goto, Disaster Prevention Research Institute, Kyoto University, Gokasho, Uji, Kyoto 611-0011, Japan. E-mail: goto@ catfish.dpri.kyoto-u.ac.jp
} 
mitigation efforts [7]. For example, rapid estimation of human and economic losses after the occurrence of earthquakes is provided by the PAGER system $[8,9]$, which utilizes the effects of amplification.

Several models of the ground amplification currently incorporated in the systems are usually represented by a single characterized value, e.g., Vs30, the average S-wave velocity down to $30 \mathrm{~m}$ depth, evaluated from the geomorphologic classification [10] and the topographic data [11, 12]. The site-specific values are estimated from the regression formula constructed by the data set of the measured values and classification at the same site. The approach is efficient enough to estimate the ground amplification globally as a first-order approximation, but the spatial resolution is not as high as the visual image of the colored maps.

More precise models of the site amplification based on the observed ground motions on horizontal or vertical arrays have been reported in many research papers $[1,13,14,15,16]$. Most of the studies identify the corresponding physical velocity structures by applying a nonlinear inversion scheme, and they are regarded as the actual velocity structures beneath the sites. One of the important problems is that the obtained results sometimes do not ensure a unique solution because of the local optimals $[13,15]$. This implies that estimated velocity structure may involve some uncertainty, and therefore more reliable and specific models are required based on the observed ground amplifications.

To deal with this problem, I propose a new series expansion of the ground amplification as the preprocessing of its modeling. The expansion is based on a sequence of simple ground transfer functions corresponding to a two-layered structure. This means that each term has a corresponding two-layered structure. The objective is to extract the major components from the amplification, and to find the contribution explicitly. The preprocessing is applicable to complex amplification shapes such as those containing multiple peaks. The representation may help to find the most reliable models corresponding to each peak and their contributions uniquely as the superposition.

This idea comes from decomposition techniques of the given functions in the Fourier domain. Several techniques have been proposed in previous studies, e.g., Cepstrum [17], modal analysis $[18,19]$, the discrete wave number method [20], eigenfunction expansion [21], etc. Zhao [19] applies a response of a single degree of freedom to the modal response of a soft-soil layer that has a straight line distribution of S-wave velocities. The model of modal response has been widely applied in stochastic modeling of the power spectrum on the ground surface since Kanai and Tajimi $[22,23,24]$. In other words, the proposed expansion based on the response of the two-layered structure provides a more physically consistent alternative to the Kanai-Tajimi spectrum because it is based on the fundamental theory of elastic wave propagation. This indicates that the series expansion may be friendly to the stochastic modeling, and each term is naturally associated with the physics model.

\section{SEQUENCE OF NORMALIZED FUNCTIONS BASED ON GROUND TRANSFER FUNCTION}

Let us consider 2D SH wave propagation in a two-layered structure, which consists of a horizontal surface layer with a thickness of $h$ and a half space basement. The S-wave velocity and density of the materials are $\beta_{1}$ and $\rho_{1}$ in the surface layer, and $\beta_{0}$ and $\rho_{0}$ in the basement. The material properties $\rho_{0}, \rho_{1}, \beta_{0}$ and $\beta_{1}$ are positive real. When an incident plane wave propagates vertically into the surface layer through the interface, the ratio of the free surface amplitude to the incident amplitude is analytically derived as follows $[25,26]$ :

$$
h_{f}(\omega)=\frac{2}{\cos \left(\omega h / \beta_{1}\right)+i r_{1,0} \sin \left(\omega h / \beta_{1}\right)},
$$

where $\omega$ is the angular frequency, $r_{1,0}$ is the impedance ratio defined by $\rho_{1} \beta_{1} / \rho_{0} \beta_{0}$, and $i$ is the imaginary unit. The ratio $h_{f}$ is usually named the ground transfer function for the two-layered structure. The ground transfer function quantifies an amplification at the particular site, and is widely applied to problems in earthquake engineering. 

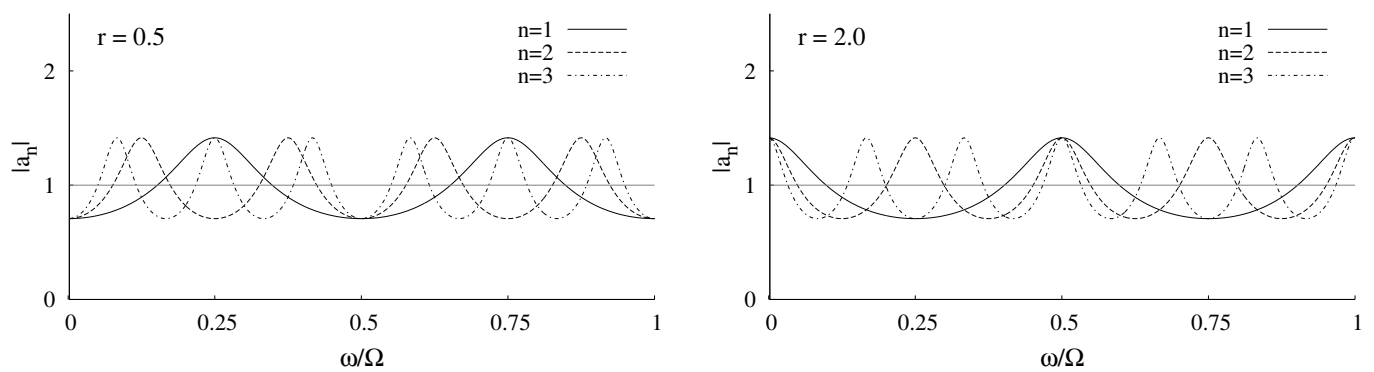

Figure 1. Function shape $\left|a_{n}\right|(n=1,2,3)$ (left: $r=0.5$, right: $\left.r=2\right)$.

Here, I define a sequence of functions $\left\{a_{n}(r, \omega) \mid n \in \mathbf{N}\right\}$ based on the ground transfer function. Let $\Omega$ be positive real, and $a_{n}(r, \omega)$ be a function; $a_{n}:(0, \infty) \times[0, \Omega] \ni(r, \omega) \rightarrow \mathbf{C}$, such that

$$
a_{n}(r, \omega) \equiv \frac{\sqrt{r}}{\cos (2 \pi n \omega / \Omega)+i r \sin (2 \pi n \omega / \Omega)} \quad(n \in \mathbf{N})
$$

Figure 1 shows examples of the function shape $\left|a_{n}\right|$ for $n=1,2$, and 3; the left panel corresponds to $r=0.5$, and the right panel to $r=2$. The number of peaks increases as $n$ increases, and their shape is controlled by the argument $r$. Obviously, $a_{n}$ becomes an exponential function $e^{-2 \pi i n \omega / \Omega}$ when $r=1$, and its absolute value keeps 1 independent of $\omega$.

Operator $\langle f\rangle$ is defined by the Lebesgue integration of $f \in L^{2}([0, \Omega], \omega / \Omega)$, as follows:

$$
\langle f\rangle \equiv \frac{1}{\Omega} \int_{[0, \Omega]} f d \omega
$$

The $L^{2}$-norm is denoted by $\|f\| \equiv \sqrt{\left\langle|f|^{2}\right\rangle}$, and the inner product of functions $f, g \in L^{2}$ is defined by the operator $\left\langle f^{*} g\right\rangle$, where $f^{*}$ denotes a complex conjugate of $f$. I introduce the norm and the inner product between the members of $\left\{a_{n}\right\}$ in order to characterize the sequences.

In the appendix, theorems 1-4 and their proofs are related to properties of the sequence of the function $\left\{a_{n}\right\}$. Theorem 1 indicates that the norm of each member of $\left\{a_{n}\right\}$ is equal to 1 . That means $a_{n}$ is normalized in $L^{2}$ space. Theorem 2 indicates that the inner product of $a_{n}$ and 1 is equal to 0 for all $n \in \mathbf{N}$.

Let $\left\{a_{n}\right\},\left\{b_{n}\right\}$ be sequences of functions $a_{n}\left(r_{a}, \omega\right)$ and $b_{n}\left(r_{b}, \omega\right)$, where $r_{a}$ and $r_{b}$ are positive real. Let $\left\{a_{n}^{*}\right\}$ be a sequence of complex conjugate functions of $a_{n}$. Theorem 3 gives a property of the inner products of $a_{m}$ and $b_{n}$, for all $m, n \in \mathbf{N}$, and theorem 4 gives a property of the inner products of $a_{m}^{*}$ and $b_{n}$. They indicate that a particular pair of $\left(a_{m}, b_{n}\right)$ or $\left(a_{m}^{*}, b_{n}\right)$ with the relation of $k+l=$ odd is orthogonal in terms of the inner product. This property does not depend on the arguments $r_{a}$ and $r_{b}$.

I discussed a similar property of the cross terms of two different transfer functions in a previous study [27]. The cross term was defined by the limit of the inner product; $\lim _{\Omega \rightarrow \infty}\left\langle h_{f}^{*} g_{f}\right\rangle$, which cannot be the inner product. The analytical discussion applied only when the ratio $h / \beta_{1}$ was a rational number that was still specific in a variety of transfer functions although they are countably infinite. The sequence of the function $\left\{a_{n}\right\}$ is also a countably infinite set, whereas a completion of the function space spanned by $\left\{a_{n}\right\}$ is applied to construct a function space. This naturally extends the discussion to the case that was excluded in the previous study. 
H. GOTO

\section{FUNCTION SPACE SPANNED BY $\left\{a_{n}\right\},\left\{a_{n}^{*}\right\}$ AND $\{1\}$}

Let $M(p, q)$ be a map; $M: \mathbf{N}^{2} \ni(p, q) \rightarrow \mathbf{N}$, such that $M(p, q)=2^{p-1}(2 q-1)$. I define a subset $\boldsymbol{a}_{p}(p \in \mathbf{N}+0)$, as follows:

$$
\begin{aligned}
& \boldsymbol{a}_{0} \equiv\{1\}, \\
& \boldsymbol{a}_{p} \equiv\left\{a_{M(p, q)} \mid q \in \mathbf{N}\right\}+\left\{a_{M(p, q)}^{*} \mid q \in \mathbf{N}\right\} \quad(p \in \mathbf{N}) .
\end{aligned}
$$

Let $\mathcal{X}\left(\boldsymbol{a}_{p}\right)(p \in \mathbf{N}+0)$ be a completion function space spanned by $\boldsymbol{a}_{p}$. An equivalence relation between $f \in L^{2}$ and $g \in \mathcal{X}\left(\boldsymbol{a}_{p}\right)$ is introduced such that $\|f-g\|=0 \Rightarrow f \sim g$. A function space $X\left(\boldsymbol{a}_{\boldsymbol{p}}\right)$ is defined by $X\left(\boldsymbol{a}_{p}\right) \equiv\left\{f \in L^{2} \mid{ }^{\exists} g \in \mathcal{X}\left(\boldsymbol{a}_{p}\right)\right.$ s.t. $\left.f \sim g\right\} . X\left(\boldsymbol{a}_{p}\right)$ is a Hilbert space.

For example, $X\left(\boldsymbol{a}_{1}\right)$ is spanned by $\boldsymbol{a}_{1}=\left\{a_{1}, a_{1}^{*}, a_{3}, a_{3}^{*}, a_{5}, a_{5}^{*}, \cdots\right\}$, and $X\left(\boldsymbol{a}_{2}\right)$ by $\boldsymbol{a}_{2}=$ $\left\{a_{2}, a_{2}^{*}, a_{6}, a_{6}^{*}, a_{10}, a_{10}^{*}, \cdots\right\}$. Each member of $\boldsymbol{a}_{p_{1}}$ and $\boldsymbol{a}_{p_{2}}$ for $p_{1} \neq p_{2}$ is not overlapped $\left(\boldsymbol{a}_{p_{1}} \cap\right.$ $\left.\boldsymbol{a}_{p_{2}}=\phi\right)$, and every $a_{n}$ belongs to any one of $\boldsymbol{a}_{p}$, because the map $M$ is a bijection between $\mathbf{N}^{2}$ and $\mathbf{N}$. The important property that characterizes the function space is orthogonality, which is derived from the property of $\left\langle a_{m}^{*} b_{n}\right\rangle$ and $\left\langle a_{m} b_{n}\right\rangle$.

Theorem 5 in the appendix indicates that each pair of the function space $X\left(\boldsymbol{a}_{p_{1}}\right)$ and $X\left(\boldsymbol{a}_{p_{2}}\right)$ $\left(p_{1} \neq p_{2}\right)$ is orthogonal. This means that a function space spanned by $\left\{a_{n}\right\}+\left\{a_{n}^{*}\right\}+\{1\}$ is a direct sum of the spaces; $\bigoplus_{p=0}^{\infty} X\left(\boldsymbol{a}_{p}\right)$, and the space is a subset of the entire function space, $L^{2}([0, \Omega], \omega / \Omega)$.

I define a function series $\left\{e_{n}\right\}$, such that $e_{n} \equiv e^{-2 \pi i n \omega / \Omega}$, which is a special case of $a_{n}$, such that all the arguments $r$ equal to 1 . A completion function space spanned by $\left\{e_{n}\right\}+\left\{e_{n}^{*}\right\}+\{1\}$ is equal to $L^{2}$ space because the Fourier series is a complete orthonormal system. Let $X_{p}^{F}(p \in \mathbf{N})$ be a completion function space spanned by $\left\{e_{M(p, q)} \mid q \in \mathbf{N}\right\}+\left\{e_{M(p, q)}^{*} \mid q \in \mathbf{N}\right\}$, and $X_{0}^{F}$ by $\{1\} . L^{2}$ is a direct sum of the spaces; $L^{2}=\bigoplus_{p=0}^{\infty} X_{p}^{F} \supseteq \bigoplus_{p=0}^{\infty} X\left(\boldsymbol{a}_{p}\right) . X\left(\boldsymbol{a}_{p_{1}}\right)$ and $X_{p_{2}}^{F}$ are orthogonal for $p_{1} \neq p_{2}$ (theorem 5). Therefore, $X_{p}^{F} \supseteq X\left(\boldsymbol{a}_{p}\right)$ for ${ }^{\forall} p \in \mathbf{N}$.

$L^{2}=\bigoplus_{p=0}^{\infty} X\left(\boldsymbol{a}_{p}\right)$ is a sufficient condition to give a series expansion with the sequences of functions to arbitrary functions in $L^{2}$ space. Theorems 6-8 in the appendix are essential in order to prove the condition. Let $r_{n}(\neq 1)(n \in \mathbf{N})$ be an argument of $a_{n}$, and $c_{2 n-1}$ be a real coefficient defined by $r_{n}$, such that

$$
c_{1} \equiv \frac{1+r_{1}}{2 \sqrt{r_{1}}}, \quad c_{2 n-1} \equiv-\sum_{m=1}^{n-1} \frac{\left\langle e_{2 m-1}^{*} a_{2 n-1}\right\rangle}{\left\langle e_{2 m-1}^{*} a_{2 m-1}\right\rangle} c_{2 m-1}(n \geq 2) .
$$

Theorem 8 indicates that $e_{1}$ has a unique series of functions belonging to $\boldsymbol{a}_{1}$, and its coefficient is $c_{2 n-1}$. Figure 2 shows an example of a series approximation of $e_{1}$ up to the order of $n=4,16$, and 64. $r$ is 0.5 for all $a_{2 n-1}$. Each coefficient $c_{2 n-1}$ is evaluated from Eq. (6). The original definition of $e_{1}$ is compared to the series approximation for both the real and imaginary parts. As guaranteed by theorem 8 , the series converges well to the original $e_{1}$ in the figure.

The series expansion corresponding to $e_{1}$ is generalized to the case $e_{n}$ for $n \in \mathbf{N}$. Theorem 9 in the appendix indicates that all the bases $e_{n}$ can be represented by a series of functions $a_{(2 m-1) n}$. This means that $e_{n}$ is a member of the function space spanned by $a_{(2 m-1) n} \cdot e_{n}^{*}$ is also represented by the complex conjugate of $a_{(2 m-1) n}$ because $c_{2 m-1}$ is a real coefficient. Therefore, all of the bases of the Fourier series can be represented by the series of $\left\{a_{n}\right\},\left\{a_{n}^{*}\right\}$, and $\{1\}$.

Theorem 10 in the appendix indicates that a function space spanned by $\left\{a_{n}\right\},\left\{a_{n}^{*}\right\}$, and $\{1\}$ is equal to $L^{2}$ space. This means that $\left\{a_{n}\right\}+\left\{a_{n}^{*}\right\}+\{1\}$ is a complete set of bases for $L^{2}$ space as with the Fourier series. This is a notable property of the function sequences, and they can be referred to bases. The Fourier series of $L^{2}$ functions converge almost everywhere [28]. Therefore, the series expansion with the bases is available for all functions in $L^{2}$ space. This also indicates that $X\left(\boldsymbol{a}_{p}\right)$ is uniquely determined independently of the argument $r$. The independence allows to be denoted by $X_{p}$ without explicitly selecting $\boldsymbol{a}_{p}$. 

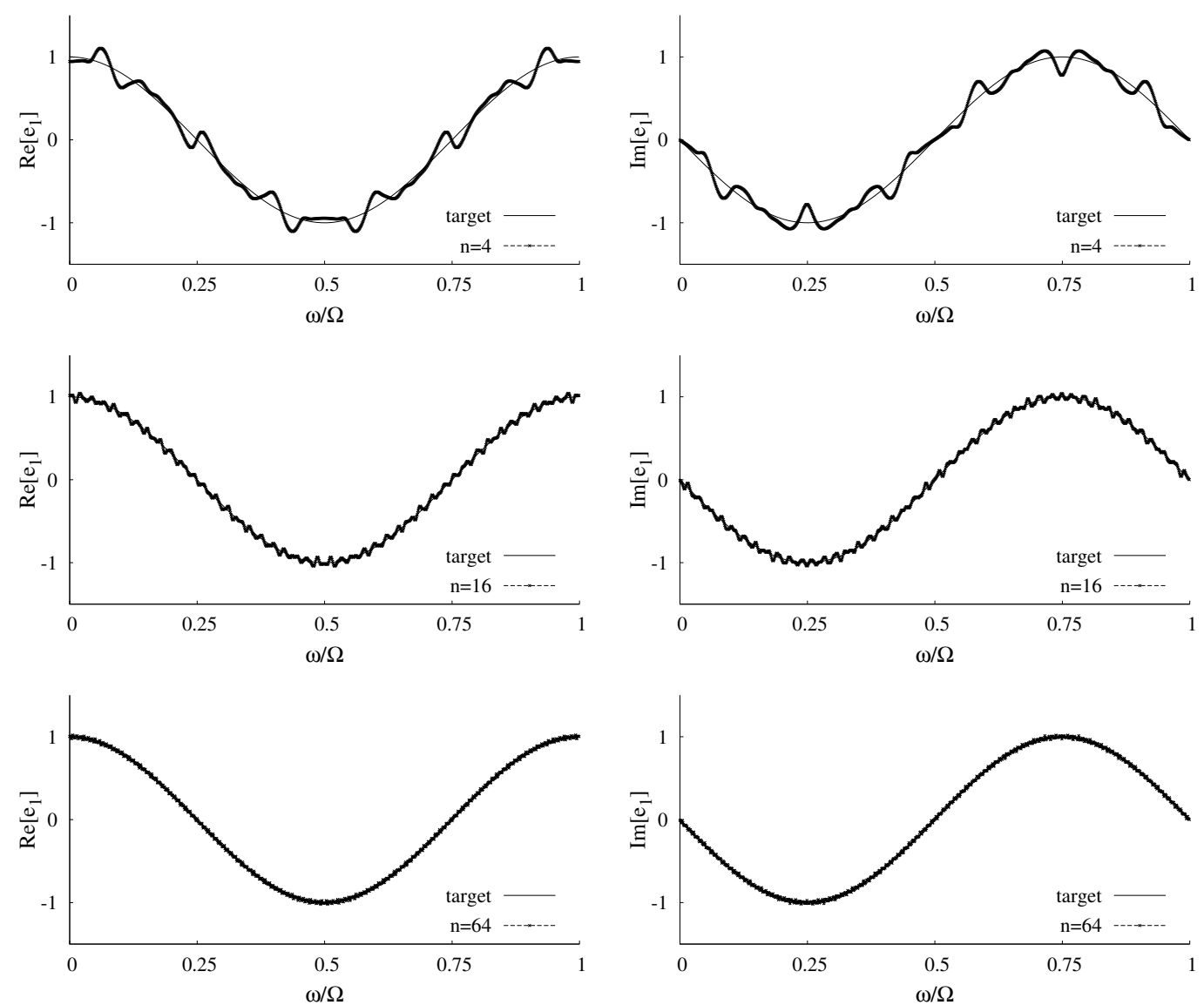

Figure 2. Comparison between $e_{1}$ and summation of $c_{2 n-1} a_{2 n-1}$ up to $n=4,16,64$ for $r=0.5$. (top: $n=4$, middle: $n=16$, bottom: $n=64$; left: real part of $e_{1}$, right: imaginary part of $e_{1}$ )

\section{SERIES EXPANSION WITH TRANSFER FUNCTION BASES}

\subsection{Coefficient of series expansion}

Let $f$ be a complex function belonging to $L^{2}$ space. The series expansion with the bases $\left\{a_{n}\right\}$ exists as proved in the previous chapter. Let $b_{n}(n \in \mathbf{N}+0)$ and $d_{m}(m \in \mathbf{N})$ be complex coefficients of the series expansion of $f$, such that

$$
f=b_{0}+\sum_{n=1}^{\infty} b_{n} a_{n}+\sum_{n=1}^{\infty} d_{n} a_{n}^{*} .
$$

The objective in this chapter is to find the value of the coefficients.

Let $F_{n}(n \in \mathbf{Z})$ be a Fourier coefficient of $f ; f=\sum_{n=-\infty}^{\infty} F_{n} e_{n}$. The basis $e_{n}$ is represented by the series of the bases $a_{n}$ as proved in theorem 9. Thus, the following series expansion is available for $f$.

$$
f=F_{0}+\sum_{n=1}^{\infty} \sum_{m=1}^{\infty} F_{n} c_{2 m-1} a_{(2 m-1) n}+\sum_{n=1}^{\infty} \sum_{m=1}^{\infty} F_{-n} c_{2 m-1} a_{(2 m-1) n}^{*} .
$$

For a given argument $r$, the expansion is unique. This is because $c_{n}$ is uniquely determined, and the Fourier coefficients are unique.

Let $P_{p}(p \in \mathbf{N}+0)$ be an orthogonal projection to $X_{p}$. Because $L^{2}$ is a direct sum of $X_{p}$, the orthogonal decomposition is available; $f=\sum_{p=0}^{\infty} P_{p} f$. Each projection is represented by a series 
of $a_{M(p, q)}$ and $a_{M(p, q)}^{*}$ as follows:

$$
P_{p} f=\sum_{q=1}^{\infty} b_{M(p, q)} a_{M(p, q)}+\sum_{q=1}^{\infty} d_{M(p, q)} a_{M(p, q)}^{*} .
$$

The inner products of $P_{p} f$ and $a_{M(p, s)}, P_{p} f$ and $a_{M(p, s)}^{*}(s \in \mathbf{N})$ are equivalent to the inner products with the original function $f$; e.g., $\left\langle a_{M(p, s)}^{*} P_{p} f\right\rangle=\left\langle a_{M(p, s)}^{*} f\right\rangle$. Then, the following system of equations for ${ }^{\forall} s \in \mathbf{N}$ is available without the projection $P_{p}$ :

$$
\begin{aligned}
\left\langle a_{M(p, s)}^{*} f\right\rangle & =\sum_{q=1}^{\infty}\left\langle a_{M(p, s)}^{*} a_{M(p, q)}\right\rangle b_{M(p, q)}+\sum_{q=1}^{\infty}\left\langle a_{M(p, s)}^{*} a_{M(p, q)}^{*}\right\rangle d_{M(p, q)} . \\
\left\langle a_{M(p, s)} f\right\rangle & =\sum_{q=1}^{\infty}\left\langle a_{M(p, s)} a_{M(p, q)}\right\rangle b_{M(p, q)}+\sum_{q=1}^{\infty}\left\langle a_{M(p, s)} a_{M(p, q)}^{*}\right\rangle d_{M(p, q)} .
\end{aligned}
$$

Let $f_{n}$ be a partial sum of the series up to $n ; f_{n}=b_{0}+\sum_{m=1}^{n}\left(b_{m} a_{m}+d_{m} a_{m}^{*}\right)$. The coefficients and the inner products satisfy the following system of equations for all $p \in \mathbf{N}+0$ :

$$
\boldsymbol{f}_{n_{p}}=\boldsymbol{A}_{p} \boldsymbol{b}_{p},
$$

where $\boldsymbol{b}_{p}$ and $\boldsymbol{f}_{n_{p}}$ are vectors consisting of the coefficients and inner product with $f_{n}$, respectively. $\boldsymbol{A}_{p}$ is a Gram matrix of $a_{M(p, q)}$. Let $q_{n}$ be the largest natural number satisfying $M\left(p, q_{n}\right) \leq n$. The vectors and the matrix are defined as follows:

$$
\begin{gathered}
\boldsymbol{b}_{p} \equiv\left\{b_{M(p, 1)}, d_{M(p, 1)}, \cdots, b_{M\left(p, q_{n}\right)}, d_{M\left(p, q_{n}\right)}\right\}^{T} \\
\boldsymbol{f}_{n_{p}} \equiv\left\{\left\langle a_{M(p, 1)}^{*} f_{n}\right\rangle,\left\langle a_{M(p, 1)} f_{n}\right\rangle, \cdots,\left\langle a_{M\left(p, q_{n}\right)}^{*} f_{n}\right\rangle,\left\langle a_{M\left(p, q_{n}\right)} f_{n}\right\rangle\right\}^{T}, \\
\left.\vdots \begin{array}{cccc}
\left\langle a_{M(p, 1)}^{*} a_{M(p, 1)}\right\rangle & \left\langle a_{M(p, 1)}^{*} a_{M(p, 1)}^{*}\right\rangle & \cdots & \left\langle a_{M(p, 1)}^{*} a_{M\left(p, q_{n}\right)}^{*}\right\rangle \\
\left\langle a_{M(p, 1)} a_{M(p, 1)}\right\rangle & \left\langle a_{M(p, 1)} a_{M(p, 1)}^{*}\right\rangle & \cdots & \left\langle a_{M(p, 1)} a_{M\left(p, q_{n}\right)}^{*}\right\rangle \\
\vdots & \vdots & \ddots & \vdots \\
\left\langle a_{M\left(p, q_{n}\right)} a_{M(p, 1)}\right\rangle & \left\langle a_{M\left(p, q_{n}\right)} a_{M(p, 1)}^{*}\right\rangle & \cdots & \left\langle a_{M\left(p, q_{n}\right)} a_{M\left(p, q_{n}\right)}^{*}\right\rangle
\end{array}\right] .
\end{gathered}
$$

Because the coefficients $b_{n}$ and $d_{n}$ are unique, $\left|\boldsymbol{A}_{p}\right|$ must be nonzero. This means that all the bases belonging to $X_{p}$ are linear independent.

Here, I define a particular finite series $\bar{f}_{n}$ up to $n$;

$$
\bar{f}_{n}=\bar{b}_{0}+\sum_{m=1}^{n} \bar{b}_{m} a_{m}+\sum_{m=1}^{n} \bar{d}_{m} a_{m}^{*},
$$

where $\bar{b}_{0}, \bar{b}_{m}$, and $\bar{d}_{m}$ are determined by minimizing the $L^{2}$-norm; $\left\|f-\bar{f}_{n}\right\| \rightarrow \min$. They satisfy the following system of equations for all $p \in \mathbf{N}+0$ :

$$
\boldsymbol{f}_{p}=\boldsymbol{A}_{p} \overline{\boldsymbol{b}}_{p}
$$

where $\overline{\boldsymbol{b}}_{p}$ and $\boldsymbol{f}_{p}$ are vectors consisting of the coefficients and inner product with $f$, respectively.

$$
\begin{gathered}
\overline{\boldsymbol{b}}_{p} \equiv\left\{\bar{b}_{M(p, 1)}, \bar{d}_{M(p, 1)}, \cdots, \bar{b}_{M\left(p, q_{n}\right)}, \bar{d}_{M\left(p, q_{n}\right)}\right\}^{T}, \\
\boldsymbol{f}_{p} \equiv\left\{\left\langle a_{M(p, 1)}^{*} f\right\rangle,\left\langle a_{M(p, 1)} f\right\rangle, \cdots,\left\langle a_{M\left(p, q_{n}\right)}^{*} f\right\rangle,\left\langle a_{M\left(p, q_{n}\right)} f\right\rangle\right\}^{T} .
\end{gathered}
$$

Because the matrix $\boldsymbol{A}_{p}$ in Eq. (17) is common to Eq. (12), the inverse matrix exists. Thus, the coefficients $\bar{b}_{n}$ and $\bar{d}_{n}$ are evaluated from $\overline{\boldsymbol{b}}_{p}=\boldsymbol{A}_{p}^{-1} \boldsymbol{f}_{p}$.

$\bar{f}_{n}$ converges in the norm, and it differs from the partial sum $f_{n}$ because $f_{n}$ converges to $f$ almost everywhere from its definition. $b_{n}$ and $d_{n}$ are directly calculated from $c_{n}$ with Eq. (6) when the 

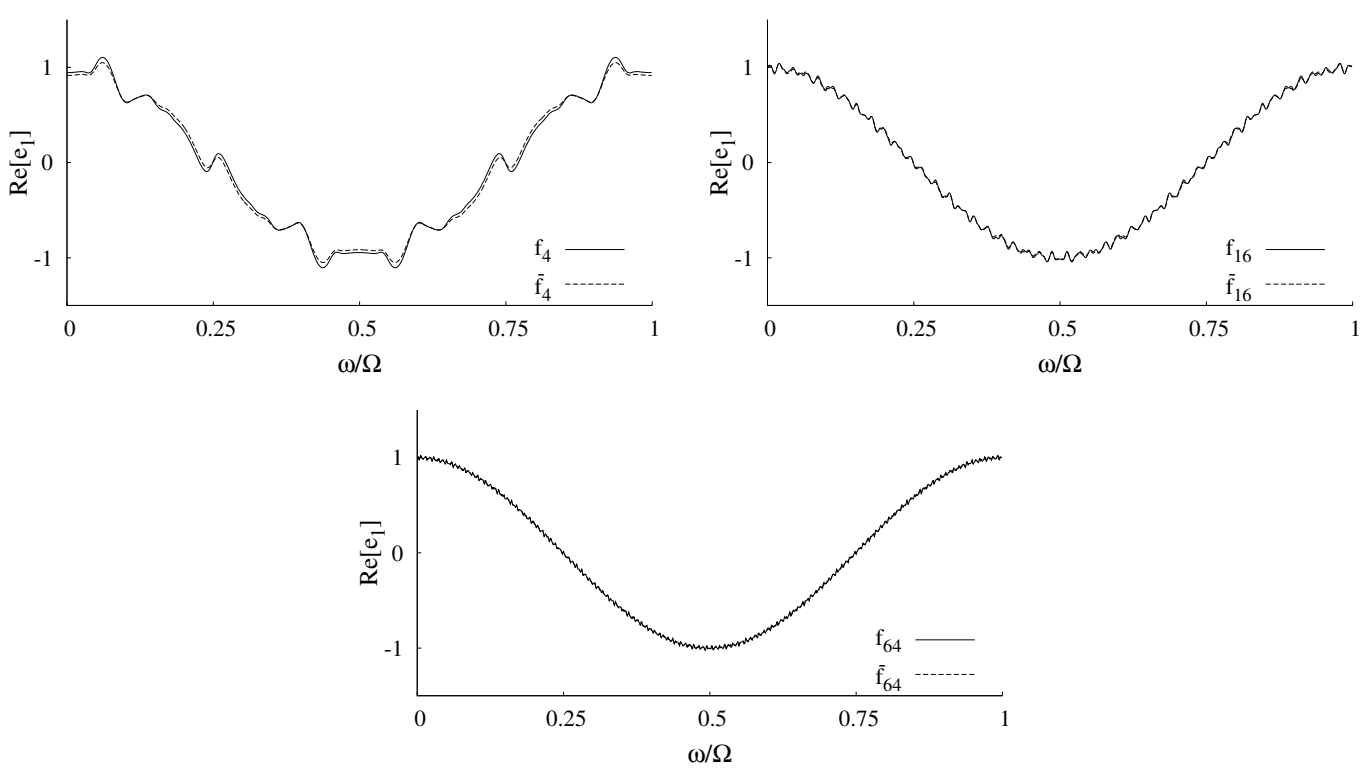

Figure 3. Comparison between partial sum $f_{n}$ and the approximation $\bar{f}_{n}$ (top left: $n=4$, top right: $n=16$, bottom: $n=64$ ).

Table I. Parameter set of Model A.

\begin{tabular}{cccc}
\hline & density $\left[\mathrm{kg} / \mathrm{m}^{3}\right]$ & S-wave velocity $[\mathrm{m} / \mathrm{s}]$ & thickness $[\mathrm{m}]$ \\
\hline surface layer \#1 & 1500 & 200 & 5.0 \\
surface layer \#2 & 1800 & 350 & 27.0 \\
\hline basement & 2000 & 1000 & - \\
\hline
\end{tabular}

Fourier coefficients of $f$ are available. For the general case, $\bar{f}_{n}$ is one of the candidates of the approximated series for $f$. Figure 3 shows the comparison between the partial sum $f_{n}$ and the approximation $\bar{f}_{n}$ for $e_{1}$. Three patterns of the number of summations $(n=4,16,64)$ are plotted in each panel. The function shape converges well to $R e\left[e_{1}\right]$ as $n$ increases, and the discrepancy between $f_{n}$ and $\bar{f}_{n}$ decreases. The rigorous proof that $\lim _{n \rightarrow \infty} \bar{f}_{n}=f$ almost everywhere may be required to approximate $f_{n}$ by $\bar{f}_{n}$, whereas I adopt the approximation to exhibit the series expansion of the target functions based on the convergence shown in Figure 3.

\subsection{Numerical experiments}

I perform numerical experiments on the series expansion for ground amplifications. The amplifications are modeled by a ground transfer function corresponding to three-layered structures, which consist of two surface layers lying over a half space basement. I artificially create two structure models, namely Model A and Model B. The detailed properties of each model are shown in Tables I and II. Internal dampings are absent throughout the materials. The target transfer functions are numerically calculated from the transfer matrix [25]. The maximum frequency, $\Omega / 2 \pi$, is $20 \mathrm{~Hz}$.

The system of equations (Eq. (17)) is numerically solved. The inner products involved in the vector $\boldsymbol{f}_{p}$ and the matrix $\boldsymbol{A}_{p}$ are calculated by numerical integration over an interval of $0.0195 \mathrm{~Hz}$; the frequency range is discretized into 1024 sections. The number of summations is $n=512$, and the argument of the basis is $r=0.5$. The sum of the effective terms is also calculated. The top five terms are selected from the absolute values of the coefficients, and they are summed up. 
H. GOTO

Table II. Parameter set of Model B.

\begin{tabular}{cccc}
\hline & density $\left[\mathrm{kg} / \mathrm{m}^{3}\right]$ & S-wave velocity $[\mathrm{m} / \mathrm{s}]$ & thickness $[\mathrm{m}]$ \\
\hline surface layer \#1 & 1500 & 180 & 20.0 \\
surface layer \#2 & 1800 & 500 & 12.0 \\
\hline basement & 2000 & 1000 & - \\
\hline
\end{tabular}

Table III. Coefficients of the top 5 terms $(r=0.5)$.

\begin{tabular}{rrrrrr}
\hline & \multicolumn{2}{c}{ Model A } & & \multicolumn{2}{c}{ Model B } \\
\cline { 2 - 3 } \cline { 5 - 6 } & basis & \multicolumn{1}{c}{ coefficient } & & basis & \multicolumn{1}{c}{ coefficient } \\
\hline$\# 1$ & $a_{2}$ & $4.2224-0.5627 i$ & & $a_{3}$ & $2.2417+3.2029 i$ \\
$\# 2$ & $a_{3}$ & $-1.7306+0.2508 i$ & & $a_{7}$ & $-2.0584+1.0492 i$ \\
$\# 3$ & $a_{7}$ & $1.4030-0.6319 i$ & & $a_{9}$ & $1.4234+0.7441 i$ \\
$\# 4$ & $a_{5}$ & $-1.0732+0.3754 i$ & & $a_{2}$ & $-0.9818-1.2507 i$ \\
$\# 5$ & $a_{11}$ & $-0.5584+0.4951 i$ & & $a_{13}$ & $0.3380+1.0907 i$ \\
\hline
\end{tabular}
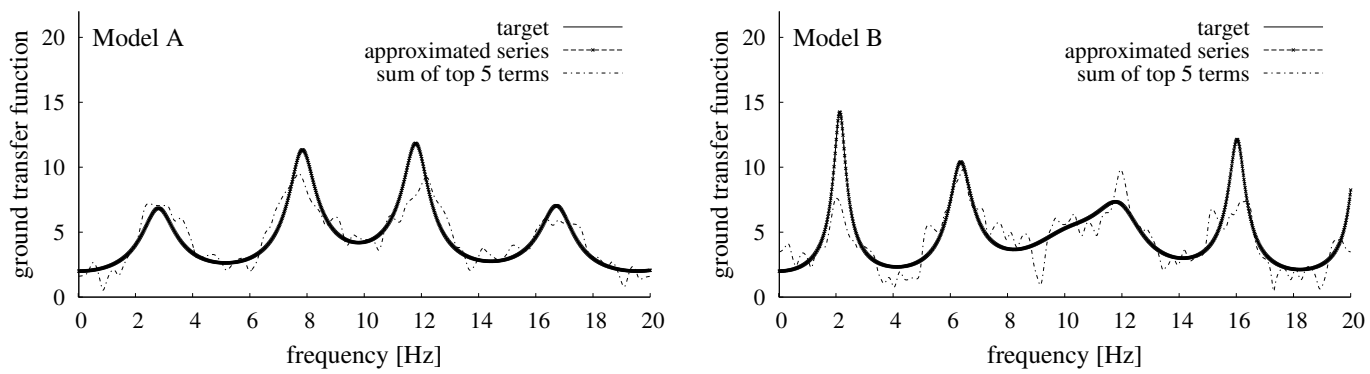

Figure 4. Comparison of absolute values among the target ground transfer function, approximated series, and sum of the top 5 terms $(r=0.5)$ (left: Model A, right: Model B).

Figure 4 shows the absolute value of the target transfer functions for Model A and Model B, respectively. They are compared to the approximated series $\bar{f}_{n}$. For both cases, the series represents the target functions well. Note that the approximation series represents the discrepancy between the values at 0 and $20 \mathrm{~Hz}$ for Model B. The figures also show the sum of the top five terms. Table III lists the coefficients of the top five terms. The summations represent some features such as the peak and valley frequencies. Figure 5 shows the absolute values of the coefficients in the order of $n$. The effective terms are allocated around the lower order of $n$, and the absolute values decrease as $n$ increases.

The lowest peak frequencies for the target functions of Model A and B are $2.8 \mathrm{~Hz}$ and $2.1 \mathrm{~Hz}$. On the other hand, the most effective bases are $a_{2}$ for Model A and $a_{3}$ for Model B, and the lowest peak frequencies are $2.5 \mathrm{~Hz}$ and $1.6 \mathrm{~Hz}$, respectively. They can give the associated transfer functions for the two-layered structure represented by Eq. (1). The parameters related to the frequency are calculated as $h / \beta_{1}=0.1 \mathrm{~s}$ and $0.15 \mathrm{~s}$, respectively. Physically, $h / \beta_{1}$ denotes the one-way travel time from the surface to the basement. Figure 6 shows the plots of the one-way travel time from the free surface to the basement against the impedance ratio. The sum of the selected terms from the series cannot generally define the associated diagram; only the single terms/bases give the associated diagram defined by Eq. (1). Therefore, the diagrams for the target models and the most effective bases are compared. This implies that the effective basis detects the important feature, the one-way travel time of the three-layered models. 

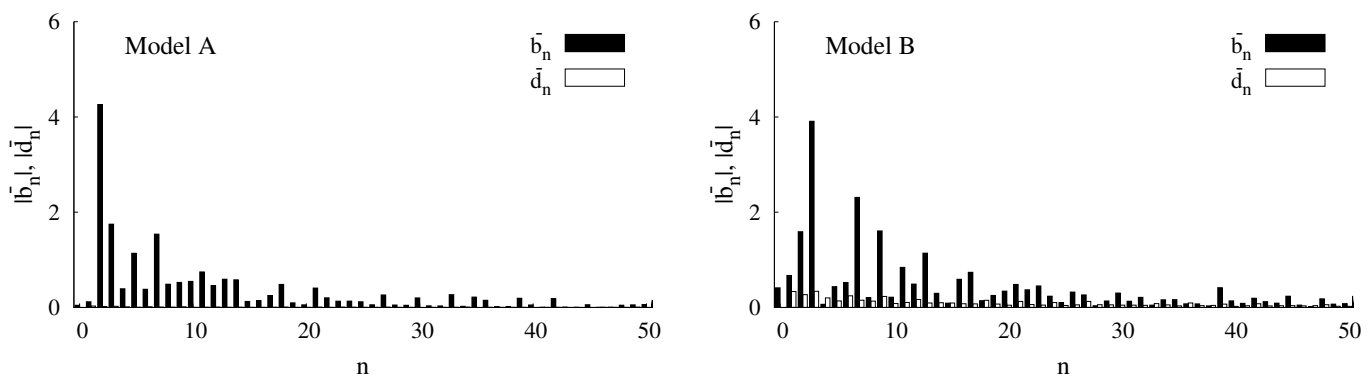

Figure 5. Absolute values of coefficients $\bar{b}_{n}$ and $\bar{d}_{n}$ (left: Model A, right: Model B).
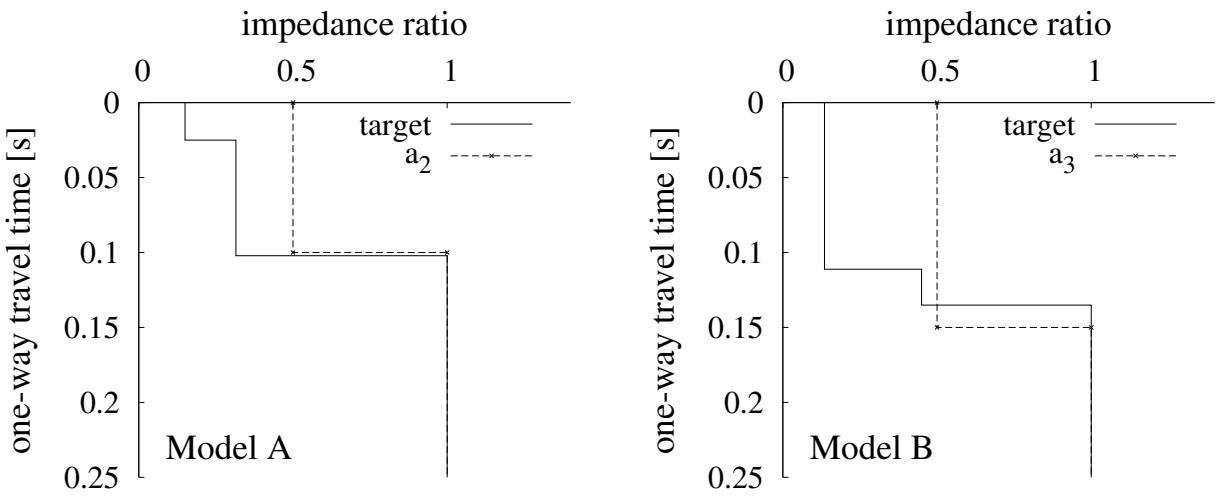

Figure 6. Plots of the one-way travel time to the basement against the impedance ratio. Comparison between target and corresponding models for the most effective basis (left: Model A, right: Model B).

\section{APPLICATION: PHYSICS-BASED DECOMPOSITION WITH SIMPLE TRANSFER FUNCTIONS}

\subsection{Decomposition procedure}

The complete set of the bases is defined when the upper bound of the domain $\Omega$ is given. The minimum frequency that will give the peak for the absolute value of the basis is $\omega_{n}=\Omega / 4 n$ for $a_{n}$. The frequency physically means the lowest resonance frequencies and the fundamental shaking mode of the two-layered structure corresponding to the basis. The maximum value of the frequencies is $\omega_{1}=\Omega / 4$. I assume that the target amplification, e.g., generated from the more complex structure, contains several peaks, and the frequencies exceed $\Omega / 4$. The series expansion exists, whereas the set of the bases cannot include the physically consistent bases corresponding to the peak frequency exceeding $\Omega / 4$. This indicates that the upper bound $\Omega$ should be assigned so that the set of the bases includes the physically consistent bases.

The argument $r$ controls the sharpness around the peaks, and it physically represents the impedance ratio between the basement and surface layers of its corresponding model. Although the series expansion is available for arbitrary values of $r$, the selection of $r$ is also essential for the physics-based series expansion. As an example, I exhibit the plots of the one-way travel time to the basement against the impedance ratio in Fig. 6. The model for the most effective bases enhances the region with material contrast, whereas the magnitude of the contrast cannot be determined only from the series expansion. Its determination requires additional criteria. 
H. GOTO

Here, I propose a technique to determine the parameters $\Omega$ and $r$, and the series expansion based on the parameters is named physics-based decomposition. I discuss its performance with some numerical examples.

In the first stage, I find out physically consistent bases that should be included in the series expansion. When there are some candidates based on prior information, e.g., when a rough velocity structure is available, they should be adopted. In the other cases, I search them by fitting the peak shapes and frequencies. Let $H_{f}(\omega)$ be a target amplification defined in the domain $\left[0, \Omega^{\prime}\right]$. I define the candidates of the bases $h_{f}^{m}(r, \omega)$, and measure the fitness $J^{m}(r)$, as follows:

$$
\begin{gathered}
h_{f}^{m}(r, \omega) \equiv \frac{\sqrt{r}}{\cos (2 \pi \omega / m d \Omega)+i r \sin (2 \pi \omega / m d \Omega)} \quad(m \in \mathbf{N}), \\
J^{m}(r) \equiv \frac{\int_{0}^{\Omega^{\prime}}\left|H_{f}(\omega)-h_{f}^{m}(r, \omega)\right|^{2} d \omega}{\sqrt{\int_{0}^{\Omega^{\prime}}\left|H_{f}(\omega)\right|^{2} d \omega} \sqrt{\int_{0}^{\Omega^{\prime}}\left|h_{f}^{m}(r, \omega)\right|^{2} d \omega}},
\end{gathered}
$$

where $d \Omega$ is positive real, and represents an interval that defines the sequence $\left\{h_{f}^{m}\right\}$.

Let $\left\{\left(m_{1}, r_{1}\right),\left(m_{2}, r_{2}\right), \cdots,\left(m_{K}, r_{K}\right)\right\}$ be parameter sets that give the local minimum of $J^{m}(r)$ in $(m, r) \in \mathbf{N} \times(0, \infty)$. $K$ denotes the number of sets selected from the local minimums. When the bases are selected from prior information, without searching the local minimums of $J^{m}(r)$, the corresponding sets of $\left(m_{k}, r_{k}\right)(k \in\{1, \cdots, K\})$ and $d \Omega$ are evaluated so that the peak frequencies and the impedance ratio are the same.

In the second stage, the upper bound of the domain $\Omega$ is determined. Let $\left\{\bar{m}_{1}, \cdots, \bar{m}_{K}\right\}$ be a coprime set so that $m_{k}=p \bar{m}_{k}$, where $p$ is a common factor $(p \in \mathbf{N}) . \Omega$ and the corresponding indexes of the bases $n_{k}$ are evaluated, as follows:

$$
\begin{aligned}
& \Omega=p \prod_{k=1}^{K} \bar{m}_{k} d \Omega, \\
& n_{k}=\frac{1}{\bar{m}_{k}} \prod_{l=1}^{K} \bar{m}_{l} .
\end{aligned}
$$

Following the representation, the complete bases $\left\{a_{n}\right\}+\left\{a_{n}^{*}\right\}+\{1\}$ for $L^{2}([0, \Omega], d \omega / \Omega)$ includes $\left\{a_{n_{1}}, \cdots, a_{n_{K}}\right\}$, and they are equal to $\left\{h_{f}^{1}, \cdots, h_{f}^{K}\right\}$, respectively. Therefore, the series expansion with the set of bases contains the physically consistent terms.

However, in reality, the upper bound $\Omega^{\prime}$ of the amplification is usually defined by some other limitations, e.g., the frequency property of the sensors, signal levels of high frequency, etc. When $\Omega$ defined by Eq. (22) exceeds $\Omega^{\prime}$, the amplification in the outer domain $\omega \in\left(\Omega^{\prime}, \Omega\right]$ is not available . If some prior information and/or physics-based suggestions are available, I can apply them to define the amplification in the domain. For general cases, a method for defining the amplification is required.

In the third stage, a regularized decomposition of the target function is found. I propose the following minimization problem to find the optimum set of coefficients $\left\{b_{0}, b_{1}, \cdots, b_{n}\right\}$ and $\left\{d_{1}, \cdots, d_{n}\right\}$

$$
\begin{gathered}
J=J_{1}+\varepsilon J_{2} \longrightarrow \min . \\
J_{1}=\frac{1}{\Omega^{\prime}} \int_{0}^{\Omega^{\prime}}\left|H_{f}(\omega)-b_{0}-\sum_{m=1}^{n} b_{m} a_{m}+\sum_{m=1}^{n} d_{m} a_{m}^{*}\right|^{2} d \omega \\
J_{2}=\left|b_{0}\right|^{2}+\sum_{m=1}^{n}\left|b_{m}\right|^{2}+\sum_{m=1}^{n}\left|d_{m}\right|^{2}-\sum_{k=1}^{K}\left|b_{n_{k}}\right|^{2} .
\end{gathered}
$$

The first term $J_{1}$ represents the residual between the target $H_{f}$ and the series defined in the domain $\left[0, \Omega^{\prime}\right]$. The second term $J_{2}$ aims to minimize the coefficients except those of physically consistent 

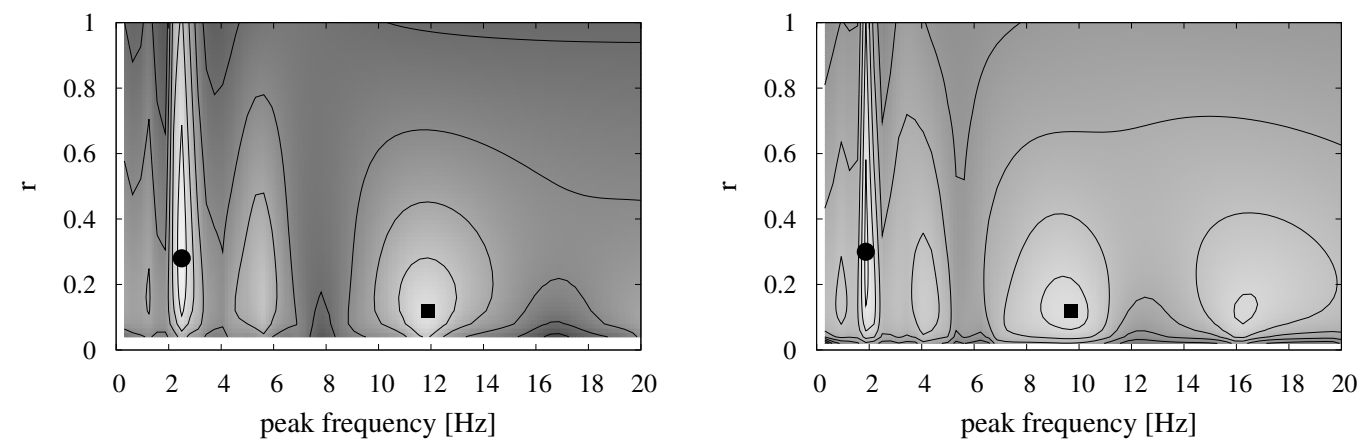

Figure 7. Contour maps of $J^{m}(r)$ (Eq. (21)). The horizontal axis represents the peak frequency of $h_{f}^{m}$. The circle and square symbols denote the locations of the first and second peaks of the local minimums, respectively (left: Model A, right: Model B).

bases, and it regularizes the ill-posedness due to the uncertainty in the outer domain. When $\Omega^{\prime}$ equals $\Omega, J_{2}$ is not required, and the minimization problem is equivalent to the convergence condition in the norm of the series expansion. $\varepsilon$ in Eq. (24) represents the weight of the contribution from $J_{1}$ and $J_{2}$. Several techniques have been proposed to identify the value of $\varepsilon$, e.g., information criteria $[29,30]$, etc. Here, I adopt the L-curve criterion [31] between $J_{1}$ and $J_{2}$, and determine the value of $\varepsilon$.

\subsection{Example 1: Application to Model A and Model B}

In order to clarify the performance of the proposed decomposition, I also apply it to Model A and Model B, which are the same models adopted in the previous section.

In the first stage, physically consistent bases are selected based on the measure $J^{m}(r) . \Omega^{\prime} / 2 \pi$ is set to $20 \mathrm{~Hz}$, the same value used in the previous examples. Figure 7 shows the contour maps of $J^{m}(r)$ with respect to the peak frequency and $r$ of $h_{f}^{m}$. A light color means smaller values of $J^{m}(r)$. Because the peak frequency $f_{\text {peak }}$ is evaluated from the index $m: f_{\text {peak }}=m d \Omega / 8 \pi$, the maps indicate the local minimums of $J^{m}(r)$ in the $(m, r)$ domain. The circle and square symbols on the map denote the location of the smallest and the next smallest peaks of the local minimums, respectively. I select the bases corresponding to the two smallest peaks for the physically consistent bases. For Model A, the peaks are estimated at $\left(f_{\text {peak }}, r\right)=(2.5 \mathrm{~Hz}, 0.28)$ and $(11.875 \mathrm{~Hz}, 0.12)$, and the corresponding $m$ values are $m_{1}=64$ and $m_{2}=304$. For Model B, the peaks are $\left(f_{\text {peak }}, r\right)=$ $(1.875 \mathrm{~Hz}, 0.3)$ and $(9.6875 \mathrm{~Hz}, 0.12)$, and the corresponding $m$ values are $m_{1}=48$ and $m_{2}=248$. $d \Omega=\Omega^{\prime} / 128 \mathrm{~Hz}$ is adopted in both cases. In the second-stage procedure, the upper bound of the domain $\Omega$ and the indexes of bases $n$ are estimated as $\Omega / 2 \pi=190 \mathrm{~Hz}, n_{1}=19$, and $n_{2}=4$ for Model $\mathrm{A}$, and $\Omega / 2 \pi=232.5 \mathrm{~Hz}, n_{1}=31$, and $n_{2}=6$ for Model B.

Based on the selected bases and the parameters, the physics-based decomposition is obtained by solving the optimization problem Eq. (24). In this decomposition, I adopt the same $r$ value for the bases belonging to the same function space $X_{p}$, and $r=1.0$ for the other bases in order to enhance the contribution from the selected bases. Figure 8 shows the L-curves that represent the trade offs between the norm $J_{1}$ and $J_{2}$ evaluated from the given values of $\varepsilon$. Based on the L-curve criterion, a suitable value of $\varepsilon$ appears at the corner of the curve. In this example, I select $\varepsilon=0.005$ for both Model A and Model B, which are located on the corner of the L-curves.

Table IV lists the coefficients for the physically consistent bases, and Fig. 9 shows the comparison between the target ground transfer functions and the function reconstructed from the sum of the two physically consistent bases. For Model A, the contribution from the first basis is about 3.4 times larger than that from the second basis in terms of the absolute value of the coefficients; for Model B, the contribution is about 4.7 times larger. If the ratio reflects the contribution of the derived two-layered model corresponding to each basis, the second basis is less effective as the 

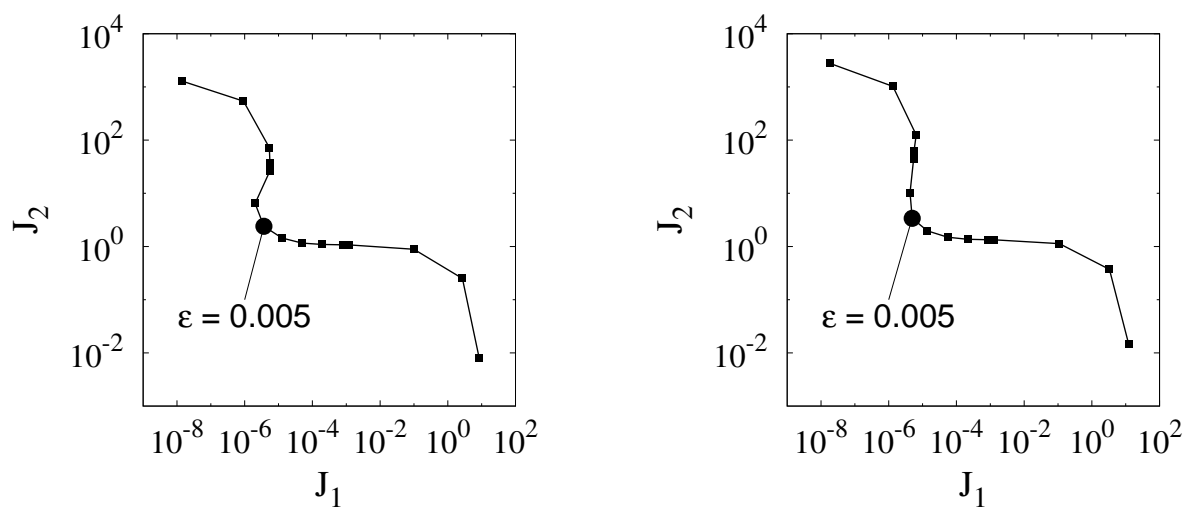

Figure 8. L-curves for the optimization problem (Eq. (24)). The circle symbols are the selected values of $\varepsilon$ according to the L-curve criterion (left: Model A, right: Model B).

Table IV. Coefficients of physically consistent bases.

\begin{tabular}{cccccccc}
\hline & \multicolumn{4}{c}{ Model A } & & \multicolumn{3}{c}{ Model B } \\
\cline { 2 - 4 } \cline { 6 - 8 } \cline { 6 - 8 } & basis & $r$ & coefficient & & basis & $r$ & coefficient \\
\hline$\# 1$ & $a_{19}$ & 0.28 & $3.8046-0.4611 i$ & & $a_{31}$ & 0.30 & $4.0568-0.2337 i$ \\
$\# 2$ & $a_{4}$ & 0.12 & $1.0836+0.3683 i$ & & $a_{6}$ & 0.12 & $0.8214-0.2764 i$ \\
\hline
\end{tabular}
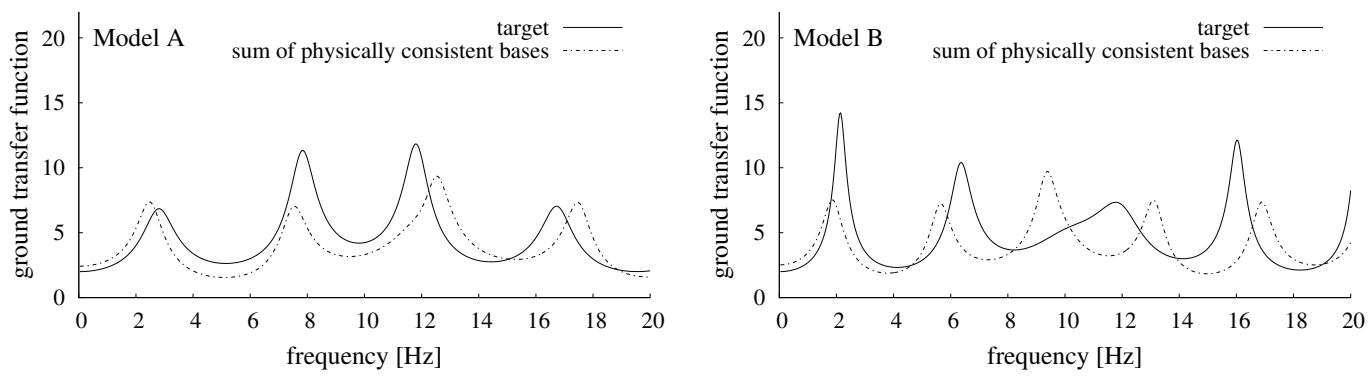

Figure 9. Comparison of absolute values between the target ground transfer function and the sum of physically consistent bases (left: Model A, right: Model B).
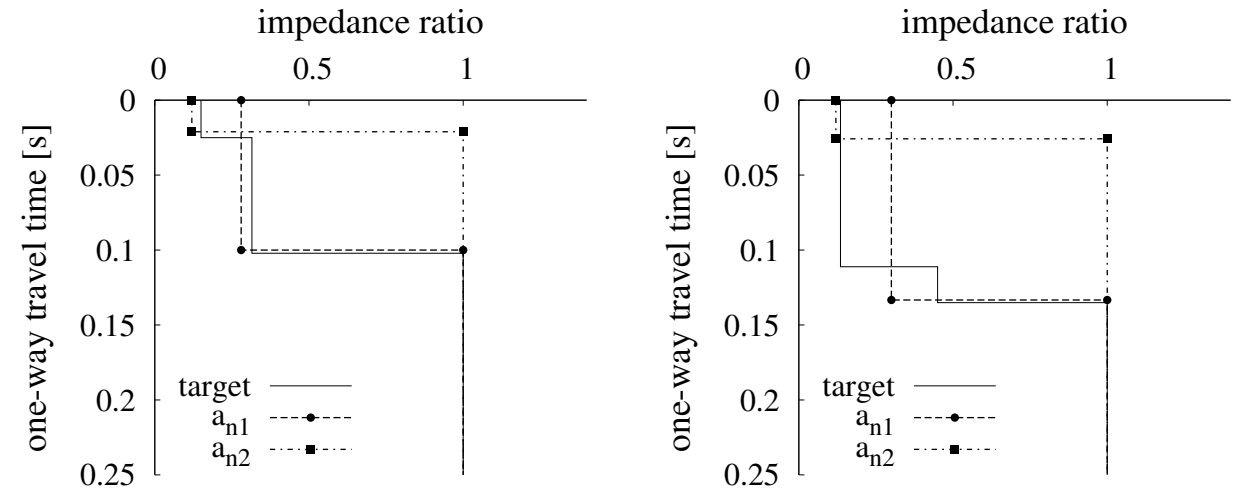

Figure 10. Plots of the one-way travel time to the basement against the impedance ratio, compared between target and corresponding models for the selected major bases (left: Model A, right: Model B). 
Table V. Parameter set of randomly generated models.

\begin{tabular}{ccccc}
\hline & density $\left[\mathrm{kg} / \mathrm{m}^{3}\right]$ & S-wave velocity $[\mathrm{m} / \mathrm{s}]$ & damping coefficient & thickness $[\mathrm{m}]$ \\
\hline surface layer \#1 & 1500 & 200 & 0.02 & 10.0 \\
surface layer \#2-\#6 & $1500-2000$ & $200-1000$ & 0.02 & variable $^{\dagger}$ \\
\hline basement & 2000 & 1000 & 0 & - \\
\hline
\end{tabular}

$\dagger$ Thickness is determined so that the one-way travel time from the basement to the top remains $0.5 \mathrm{~s}$.
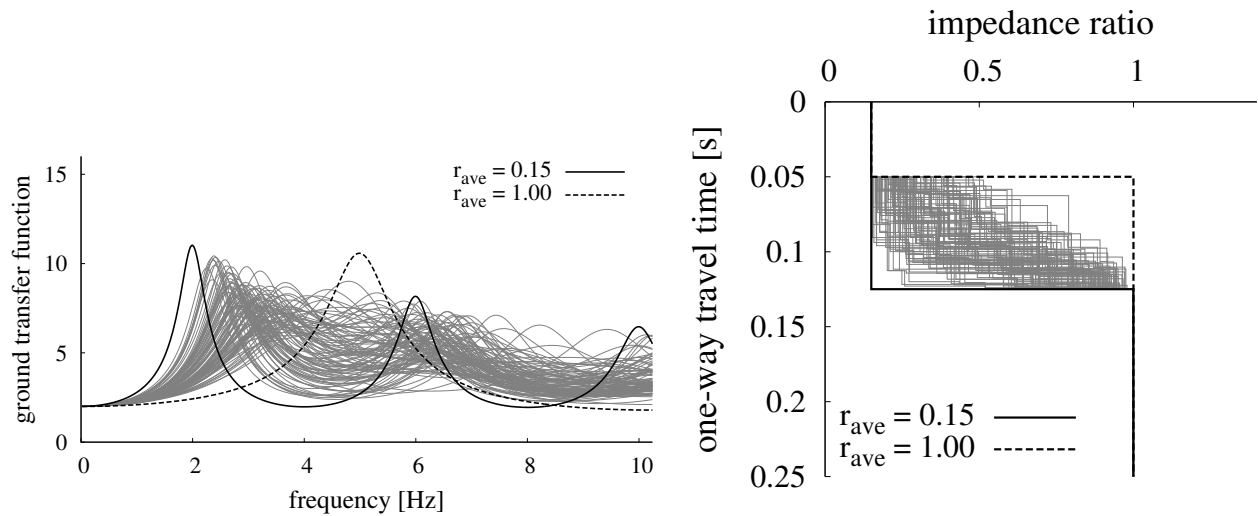

Figure 11. Target ground transfer functions (left) and plots of the one-way travel time against the impedance ratio (right) for randomly generated 100 models. Solid and dotted lines labeled $r_{\text {ave }}=0.15$ and 1.00 are for the extreme cases.

target transfer function for Model B. Although representations of the target transfer functions are not precise, it is noteworthy that they are constructed from the summation of only two simple transfer functions. The lowest peak frequencies, which are features that characterize the amplification, are well approximated in both cases.

Figure 10 shows the plots of the one-way travel time to the basement against the impedance ratio, and they are compared to the two-layered models corresponding to the selected bases. For Model A, the first basis represents the material boundary on the basement well, and the second basis also represents it well between the first and second layers. The estimated impedance ratio is correlated well with the target impedance ratio in the surface layers. For Model B, the first basis represents the material boundary on the basement well. On the other hand, the second basis is not directly related to the material boundary. As described above, the contribution from the second basis is less than in the case of Model A in terms of the coefficients, which may explain the results.

In Figure 7, the contour map for Model B indicates another candidate for the second smallest peaks at $f_{\text {peak }}=16.25 \mathrm{~Hz}$, instead of $f_{\text {peak }}=9.6875 \mathrm{~Hz}$, as the selected bases. I identified the coefficients of the bases with the same procedure, and compared the transfer functions and the plots of the one-way travel times in Figs. 9-10. The results are almost the same as for the original selected bases. This implies that the selections of the local minimums from the contour maps may not be essential if the peak values are similar, though verifying this requires a more precise investigation.

\subsection{Example 2: Application to quantification of model similarity}

I assume two-layered models, M1 and M2, with the same density and S-wave velocities for both the surface layer and the basement, but with different layer thicknesses of the layer, $h_{1}$ and $h_{2}$ $\left(h_{1}>h_{2}\right)$. I also assume another multi-layer model, M, with the same physical constants above $h_{2}$ and below $h_{1}$ with M1 and M2, and with arbitrary values within the depth from $h_{2}$ to $h_{1}$. M1 and $\mathrm{M} 2$ belong to the set of $\mathrm{M}$, whereas the ground transfer functions corresponding to M1, M2, and 


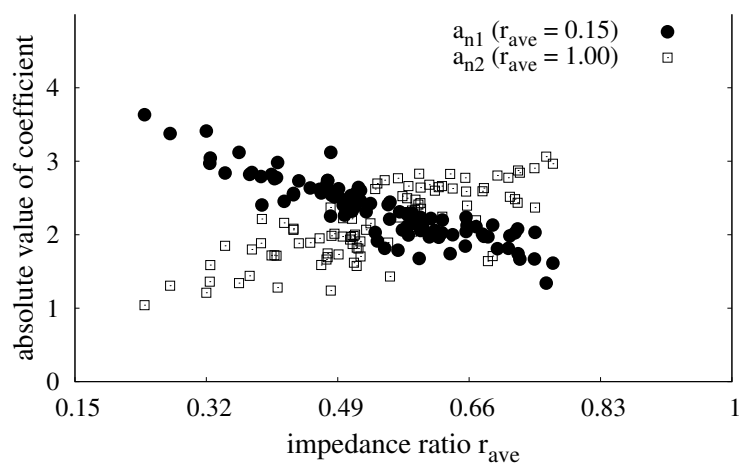

Figure 12. Absolute value of coefficients for the selected bases plotted against the impedance ratio of their average within the intermediate surface layers.

M do not generally coincide. Here, I want to quantify the similarity of M to M1 and M2. In the previous example, the coefficient of the bases for the series expansion was related to its contribution to the amplification. Therefore, quantification of the similarity from their ground transfer functions by using the series expansion may be possible. In this example, I checked the availability of the series expansion via randomly generated multi-layered models.

The model consists of six surface layers lying over a half space basement. The detailed properties are shown in Table V. The density and S-wave velocity of the intermediate surface layers \#2-\#6 are randomly generated through a uniform distribution in the range $1500-2000 \mathrm{~kg} / \mathrm{m}^{3}$ for the density and $200-1000 \mathrm{~m} / \mathrm{s}$ for the S-wave velocity. The density and velocity are sorted in ascending order from the top. The thickness of each layer is determined so that the one-way travel time from the basement to the top remains $0.125 \mathrm{~s}$. In this example, internal damping is considered in the surface layers.

The models are characterised by a single index $r_{\text {ave }}$ defined by the weighted average of the impedance ratio over the intermediate layers \#2-\#6, whose weight corresponds to the thickness of each layer. The lower extreme case that takes the lowest values is referred to as $r_{\text {ave }}=0.15$, and the higher case is referred to as $r_{\text {ave }}=1.00$. Both these extreme cases correspond to a twolayered model with different depths to the basement, $25 \mathrm{~m}$ for $r_{\text {ave }}=0.15$ and $10 \mathrm{~m}$ for $r_{\text {ave }}=1.00$. Therefore, I adopt the two transfer functions excluding the internal damping for the physically consistent bases $a_{n_{1}}$ and $a_{n_{2}}$, and calculate their contributions for the randomly generated models.

I generate 100 random models, and calculate the amplification based on the models. Figure 11 shows the amplifications and models represented by the plot between the one-way travel time to the basement and the impedance ratio. The amplifications and models for the extreme cases $r_{\text {ave }}=0.15$ and 1.00 are also plotted. The randomly generated models have a variety of impedance ratios in the range $0.05-0.125 \mathrm{~s}$ for the one-way travel time, while the extreme cases give their upper and lower bounds. The amplifications calculated from the models vary greatly in amplitude and peak frequency.

Figure 12 shows the absolute values of the coefficients for the selected bases $a_{n_{1}}$ and $a_{n_{2}}$, which correspond to the lowest $\left(r_{\text {ave }}=0.15\right)$ and highest $\left(r_{\text {ave }}=1.00\right)$ extreme cases. Each result plots the coefficients against the impedance ratio $r_{\text {ave }}$. The results show a good correlation between the impedance ratio and the coefficients. As the impedance ratio increases, the absolute values of the coefficients for $a_{n_{1}}$ decrease, and those for $a_{n_{2}}$ increase. This indicates that the contribution represented by the coefficients of the series expansion related to the similarity of the model in terms of the impedance ratio. It enhances the physical meanings of the series expansion based on the physically consistent bases. 


\section{CONCLUSION}

A sequence of functions $\left\{a_{n}\right\}$ is defined based on a simple ground transfer function of a two-layered structure. A completion function space spanned by $\left\{a_{n}\right\},\left\{a_{n}^{*}\right\}$, and $\{1\}\left({ }^{\forall} n \in \mathbf{N}\right)$ is mathematically proved to be equal to $L^{2}$ space. This means that the functions are the normal bases in $L^{2}$ space, and that all functions belonging to $L^{2}$ space have a unique series expansion. This ensures that the expansion can be used to preprocess an arbitrary ground amplification. The basis has an argument $r$. For $r=1$, they are equal to the bases of the Fourier series. This indicates that the basis is a natural extension of the Fourier bases, although its orthogonality only exists between the bases of $X_{p_{1}}$ and $X_{p_{2}}$. Numerical experiments show that the series expansion extracts the important features of the target amplification.

In practice, the expansion requires the observed ground amplification. It is directly observable from the spectral ratio of the Fourier spectra at the target site to that at a reference rock site [13]. When the observations are available, the expansion is applicable even for the site response including a 3D basin effect as the preprocessing, whereas it requires a more precise investigation of what the extracted components physically means for the general cases.

I apply the series expansion to the physics-based decomposition of the amplification. The results indicate that the contribution from the given bases can be represented by the absolute value of their coefficients. The contribution may enable direct quantification of the similarity of models. This property potentially has wide applications, e.g., spatial interpolation of the amplifications from the sites where they are reliably determined, stochastic modeling of the amplification as a mixed state of the fundamental simple states, etc. The detailed application is currently under way.

\section{ACKNOWLEDGEMENT}

I thank Prof. Hitoshi Morikawa and many other researchers associated with me for useful discussions that helped achieve the results presented in this paper.

\section{REFERENCES}

1. Kawase H. Site effects on strong ground motions. International Handbook of Earthquake and Engineering Seismology Part B. Academic Press, 2003; 1013-1030. DOI: 10.1016/S0074-6142(03)80175-4.

2. Kawase $\mathrm{H}$. The cause of the damage belt in Kobe: "the basin-edge effect," constructive interference of the direct S-wave with the basin-induced diffracted/Rayleigh waves. Seism. Res. Lett. 1996; 67(5): 25-34. DOI: 10.1785/gssrl.67.5.25.

3. Goto H, Sawada S, Morikawa H, Kiku H, Ozalaybey S. Modeling of 3D subsurface structure and numerical simulation of strong ground motion in the Adapazari basin during the 1999 Kocaeli earthquake, Turkey. Bull. Seism. Soc. Am. 2005; 95(6): 2197-2215. DOI: 10.1785/0120050002.

4. Goto H, Hata Y, Kuwata Y, Yamamoto H, Morikawa H, Kataoka S. Earthquake source and ground motion characteristics in eastern Japan during the 2011 off the Pacific coast of Tohoku earthquake. Journal of JSCE 2013; 1(1): 329-342. DOI:10.2208/journalofjsce.1.1_329.

5. Goto H, Morikawa H, Inatani M, Ogura Y, Tokue S, Zhang XR, Iwasaki M, Araki M, Sawada S, Zerva A. Very dense seismic array observations in Furukawa district, Japan. Seism. Res. Lett. 2012; 83(5): 765-774. DOI: $10.1785 / 0220120044$.

6. Silva V, Crowley H, Pagani M, Monelli D, Pinho R. Development of the OpenQuake engine, the Global Earthquake Model's open-source software for seismic risk assessment. Nat. Hazards 2013. DOI: 10.1007/s11069-013-0618-x.

7. Wein A, Johnson L, Bernknopfa R. Recovering from the ShakeOut earthquake. Earthquake Spectra 2011; 27(2): 521-538. DOI: 10.1193/1.3581225.

8. Allen TI, Marano KD, Earle PS, Wald DJ. PAGER-CAT: A composite earthquake catalog for calibrating global fatality models. Seism. Res. Lett. 2009; 80(1): 57-62. DOI: 10.1785/gssrl.80.1.57.

9. Jaiswal K, Wald DJ. Estimating economic losses from earthquakes using an empirical approach. Earthquake Spectra 2013; 29(1): 309-324. DOI: 10.1193/1.4000104.

10. Wakamatsu K, Matsuoka M. Development of the 7.5-arc-second engineering geomorphologic classification database and its application to seismic microzoning. Bull. Earthq. Res. Insts., Univ. Tokyo 2006; 81: 317-324.

11. Wald DJ, Allen TI. Topographic slope as a proxy for seismic site conditions and amplification. Bull. Seism. Soc. Am. 2007; 97(5): 1379-1395. DOI: 10.1785/0120060267.

12. Allen TI, Wald DJ. On the use of high-resolution topographic data as a proxy for seismic site conditions (Vs30). Bull. Seism. Soc. Am. 2009; 99(2A): 935-943. DOI: 10.1785/0120080255.

13. Goto H, Kawamura Y, Sawada S, Akazawa T. Direct estimation of near-surface damping based on normalized energy density. Geophys. J. Int. 2013; 194(1): 488-498. DOI: 10.1093/gji/ggt104. 
14. Seale SH, Archuleta RJ. Site amplification and attenuation of strong ground motion. Bull. Seism. Soc. Am. 1989; 79(6): 1673-1696.

15. Kurita T, Matsui K. Sensitivity analysis on measurement noise in the identification of soil properties from vertical array observation data. Earthquake Engng. Struct. Dyn. 1997; 26(9): 951-965. DOI: 10.1002/(SICI)10969845(199709)26:9;951::AID-EQE687;3.0.CO;2-H.

16. Yamada $\mathrm{K}$. Horike M. Inference of $\mathrm{Q}$-values below $1 \mathrm{~Hz}$ from borehole and surface data in the Osaka basin by three-component waveform fitting. Bull. Seism. Soc. Am. 2007; 97(4): 1267-1278. DOI: 10.1785/0120040199.

17. Bogert BP, Hearly MJR, Turkey JW. The quefrency analysis of time series for echoes: Cepstrum, pseudoautocovariance, cross-cepstrum, and saphe cracking. Proc. Symp. Time Series Analysis 1963; 209-243.

18. Dobry R, Oweis I, Urzua A. Simplified procedures for estimating the fundamental period of a soil profile. Bull. Seism. Soc. Am. 1976; 66(4): 1293-1321.

19. Zhao JX. Estimating modal parameters for a simple soft-soil site having a linear distribution of shear wave velocity with depth. Earth. Engng. Struct. Dyn. 1996; 25(2): 163-178. DOI: 10.1002/(SICI)10969845(199602)25:2;163::AID-EQE544;3.0.CO;2-8.

20. Bouchon M, Aki K. Discrete wave number representation of seismic source wave fields. Bull. Seism. Soc. Am. 1977; 67(2): 259-277.

21. Tohei T. Compete eigenfunction expansion form of the Green's function for elastic layered half-space. Arch. Appl. Mech. 2002; 72(1): 13-38. DOI: 10.1007/s004190100193.

22. Kanai K. Semi-empirical formula for the seismic characteristics of the ground. Bull. Earthq. Res. Inst. Univ. Tokyo 1957; 35(2): 309-325.

23. Tajimi H. A statistical method of determining the maximum response of a building structure during an earthquake. Proc. 2nd World Conf. Erthq. Engng. 1960; 781-797.

24. Zerva A. Spatial Variation of Seismic Ground Motions. CRC Press: Boca Raton, 2009.

25. Haskell NA. Crustal reflection of plane SH waves. J. Geophys. Res. 1960; 65(12): 4147-4150. DOI: 10.1029/JZ065i012p04147.

26. Goto H, Sawada S, Hirai T. Conserved quantity of elastic waves in multi-layered media: 2D SH case - normalized energy density. Wave Motion 2011; 48(7): 602-612. DOI: 10.1016/j.wavemoti.2011.04.011.

27. Goto H. Fundamental property of cross terms of ground transfer function. J. Appl. Mech. JSCE 2013; 69: I_435-446 (in Japanese with English abstract).

28. Carleson L. On convergence and growth of partial sums of Fourier series. Acta. Math. 1966; 116(1): 135-157. DOI: $10.1007 / \mathrm{BF} 02392815$.

29. Akaike H. On the use of the predictive likelihood of a Gaussian model. Ann. Inst. Statist. Math. 1980; 32: 311-324.

30. Kitagawa G, Gersch W. A smoothness priors-state space modeling of time series with trend and seasonality. $J$. Amer. Statist. Assoc. 1984; 79: 378-389.

31. Hansen PC. Analysis of discrete ill-posed problems by means of the L-curve. SIAM Review 1992; 34(4): 561-580.

\section{APPENDIX A. THEOREMS AND PROOFS}

Theorem 1

${ }_{n} \in \mathbf{N} ;\left\|a_{n}\right\|=1$.

Proof

${ }^{\forall} \in \mathbf{N}$;

$$
\left\langle\left|a_{n}\right|^{2}\right\rangle=\frac{1}{\Omega} \int_{0}^{\Omega} \frac{r}{\cos ^{2}(2 \pi n \omega / \Omega)+r^{2} \sin ^{2}(2 \pi n \omega / \Omega)} d \omega=\frac{1}{2 \pi} \int_{0}^{2 \pi} \frac{r}{\cos ^{2} \tilde{\omega}+r^{2} \sin ^{2} \tilde{\omega}} d \tilde{\omega}=1,
$$

where $\tilde{\omega}=2 \pi n \omega / \Omega$. Thus, $\left\|a_{n}\right\|=\sqrt{\left\langle\left|a_{n}\right|^{2}\right\rangle}=1$.

Theorem 2

${ }_{n} \in \mathbf{N} ;\left\langle a_{n}\right\rangle=0$.

Proof

${ }^{\forall} \in \mathbf{N}$;

$$
\left\langle a_{n}\right\rangle=\frac{1}{\Omega} \int_{0}^{\Omega} \frac{\sqrt{r}}{\cos (2 \pi n \omega / \Omega)+i r \sin (2 \pi n \omega / \Omega)} d \omega=\frac{1}{2 \pi} \int_{0}^{2 \pi} \frac{\sqrt{r}}{\cos \tilde{\omega}+i r \sin \tilde{\omega}} d \tilde{\omega} .
$$

For $r=1,\left\langle a_{n}\right\rangle=0$. For $r \neq 1$, let $z$ be a complex $e^{i \tilde{\omega}}$ located on a unit circle $C$ on the complex plane. The above integral is allowed to be a contour integral along $C$.

$$
\left\langle a_{n}\right\rangle=\frac{1}{2 \pi i} \oint_{C} \frac{\frac{2 \sqrt{r}}{1+r}}{z^{2}+\frac{1-r}{1+r}} d z .
$$


The poles of the integrand are located at $\pm i \sqrt{1-r / 1+r}(r<1)$, or $\pm \sqrt{r-1 / 1+r}(r>1)$. The residues corresponding to the poles have the same absolute value and opposite signs, so the integration vanishes. Then, $\left\langle a_{n}\right\rangle=0$.

Theorem 3

${ }^{\forall}, n \in \mathbf{N} ;\left\langle a_{m}^{*} b_{n}\right\rangle=0$ for $k+l=$ odd, s.t. $(k, l)$ are a coprime set of $(m, n)$.

Proof

${ }^{\exists} p \in \mathbf{N}$, s.t. $m=p k$ and $n=p l$. Let $z_{p}$ be a complex $e^{2 \pi i p \omega / \Omega} \cdot\left\langle a_{m}^{*} b_{n}\right\rangle$ is represented by a contour integral along the unit circle $C$, as follows:

$$
\left\langle a_{m}^{*} b_{n}\right\rangle=\frac{1}{2 \pi i} \oint_{C} \frac{2 \sqrt{r_{a}}}{\left(1-r_{a}\right) z_{p}^{k}+\left(1+r_{a}\right) z_{p}^{-k}} \frac{2 \sqrt{r_{b}}}{\left(1+r_{b}\right) z_{p}^{l}+\left(1-r_{b}\right) z_{p}^{-l}} z_{p}^{-1} d z_{p} .
$$

Let $S\left(z_{p}\right)$ be an integrand of Eq. (30).

1. $r_{a}=r_{b}=1$.

$$
S\left(z_{p}\right)=z_{p}^{k-l-1} \text {. }
$$

The contour integral, Eq. (30), gives 1 only for the case $k=l$, and 0 for the other cases. $k$ and $l$ should be 1 for $k=l$ because they are a coprime set. Thus, $\left\langle a_{m}^{*} b_{n}\right\rangle=0$ for $k \neq l$, and $\left\langle a_{m}^{*} b_{n}\right\rangle=1$ for $k=l=1$.

2. $r_{a}=1, r_{b} \neq 1$.

$$
S\left(z_{p}\right)=\frac{\frac{2 \sqrt{r_{b}}}{1+r_{b}}}{z_{p}^{2 l}+\frac{1-r_{b}}{1+r_{b}}} z_{p}^{k+l-1}
$$

Poles are located at $z_{p}=w_{q}^{+}$or $w_{q}^{-}$, such that

$$
\begin{aligned}
& w_{q}^{+}=\left(\frac{r_{b}-1}{1+r_{b}}\right)^{1 / 2 l} e^{\pi i q / l} \text { for } r_{b}>1, \\
& w_{q}^{-}=\left(\frac{1-r_{b}}{1+r_{b}}\right)^{1 / 2 l} e^{\pi i(2 q-1) / 2 l} \text { for } r_{b}<1
\end{aligned}
$$

where $q \in\{1, \cdots, 2 l\} .{ }^{\forall} q \in\{1, \cdots, l\} ; w_{q+l}^{ \pm}=-w_{q}^{ \pm}$. The residues corresponding to $w_{q}^{+}$and $w_{q}^{-}$ are represented as follows,

$$
\operatorname{Res}_{z_{p}=w_{q}^{ \pm}}\left[S\left(z_{p}\right)\right]=-\frac{\sqrt{r_{b}}}{l\left(1-r_{b}\right)} w_{q}^{ \pm}{ }^{k+l}
$$

For $k+l=$ odd, the sum of the residues corresponding to $w_{q}^{ \pm}$and $w_{q+l}^{ \pm}$vanishes. Thus, $\left\langle a_{m}^{*} b_{n}\right\rangle=0$ for $k+l=$ odd, and $\left\langle a_{m}^{*} b_{n}\right\rangle=-2 \sqrt{r_{b}} / l\left(1-r_{b}\right) \sum_{q=1}^{l} w_{q}^{ \pm+l}$ for $k+l=$ even. The sum is nonzero only for the case $(k, l)=(2 s-1,1)$ for all $s \in \mathbf{N}$, and then

$$
\left\langle a_{m}^{*} b_{n}\right\rangle=-\frac{2 \sqrt{r_{b}}}{1-r_{b}}\left(\frac{r_{b}-1}{1+r_{b}}\right)^{s} .
$$

3. $r_{a} \neq 1, r_{b}=1$.

$$
S\left(z_{p}\right)=\frac{\frac{2 \sqrt{r_{a}}}{1-r_{a}}}{z_{p}^{2 k}+\frac{1+r_{a}}{1-r_{a}}} z_{p}^{k-l-1} .
$$

The poles inside the unit circle $C$ are located at $z_{p}=0$ for $k \leq l$. The following power series converges because of $\left|1-r_{a} / 1+r_{a}\right|<1$.

$$
z_{p}^{-k+l+1} S\left(z_{p}\right)=\frac{2 \sqrt{r_{a}}}{1+r_{a}} \sum_{q=0}^{\infty}\left(\frac{r_{a}-1}{1+r_{a}}\right)^{q} z_{p}^{2 k q} .
$$

The $(l-k)$ th order differential of $z_{p}^{-k+l+1} S\left(z_{p}\right)$ is derived, as follows:

$$
\frac{d^{l-k}}{d z_{p}^{l-k}}\left[z_{p}^{-k+l+1} S\left(z_{p}\right)\right]=\frac{2 \sqrt{r_{a}}}{1+r_{a}} \sum_{q>[(l-k) / 2 k]} \frac{(2 k q) !}{(2 k q+k-l) !}\left(\frac{r_{a}-1}{1+r_{a}}\right)^{q} z_{p}^{2 k q+k-l}
$$


Then, residue of $S\left(z_{p}\right)$ is nonzero only for the case $(l-k) / 2 k \in \mathbf{N}+0$. That is $l=(2 s-1) k$ for all $s \in \mathbf{N}$. Thus, $\left\langle a_{m}^{*} b_{n}\right\rangle=2(l-1) ! \sqrt{r_{a}} /\left(1+r_{a}\right)\left(r_{a}-1 / 1+r_{a}\right)^{(l-1) / 2}$ only for $(k, l)=(1,2 s-1)$ $(s \in \mathbf{N})$, and $\left\langle a_{m}^{*} b_{n}\right\rangle=0$ for the other cases.

4. $r_{a} \neq 1, r_{b} \neq 1$.

$$
S\left(z_{p}\right)=\frac{\frac{4 \sqrt{r_{a} r_{b}}}{\left(1-r_{a}\right)\left(1+r_{b}\right)}}{\left(z_{p}^{2 k}+\frac{1+r_{a}}{1-r_{a}}\right)\left(z_{p}^{2 l}+\frac{1-r_{b}}{1+r_{b}}\right)} z_{p}^{k+l-1} .
$$

The poles inside the unit circle $C$ are located at $z_{p}=w_{q}^{+}$or $w_{q}^{-}$defined by Eqs. (33)-(34). The residue corresponding to $w_{q}^{ \pm}$is

$$
\operatorname{Res}_{z_{p}=w_{q}^{ \pm}}\left[S\left(z_{p}\right)\right]=-\frac{2 \sqrt{r_{a} r_{b}}}{l\left(1-r_{a}\right)\left(1-r_{b}\right)} \frac{w_{q}^{ \pm k+l}}{w_{q}^{ \pm 2 k}+\frac{1+r_{a}}{1-r_{a}}} .
$$

${ }^{\forall} q \in\{1, \cdots, l\} ; w_{q+l}^{ \pm}{ }^{2 k}=w_{q}^{ \pm 2 k}$. For $k+l=$ odd, the sum of the residues corresponding to $w_{q}^{ \pm}$and $w_{q+l}^{ \pm}$vanishes. Thus, $\left\langle a_{m}^{*} b_{n}\right\rangle=0$ for $k+l=$ odd, and

$$
\left\langle a_{m}^{*} b_{n}\right\rangle=-\frac{4 \sqrt{r_{a} r_{b}}}{l\left(1-r_{b}\right)} \sum_{q=1}^{l} \frac{w_{q}^{ \pm}{ }^{k+l}}{\left(1-r_{a}\right) w_{q}^{ \pm^{2 k}}+1+r_{a}}
$$

for $k+l=$ even.

From the results in all the cases $1-4,\left\langle a_{m}^{*} b_{n}\right\rangle=0$ for $k+l=$ odd.

\section{Theorem 4}

${ }^{\forall}, n \in \mathbf{N} ;\left\langle a_{m} b_{n}\right\rangle=0$ for $k+l=$ odd, s.t. $(k, l)$ are a coprime set of $(m, n)$.

\section{Proof}

${ }^{\exists} p \in \mathbf{N}$, s.t. $m=p k$ and $n=p l$. Let $z_{p}$ be a complex $e^{2 \pi i p \omega / \Omega} \cdot\left\langle a_{m} b_{n}\right\rangle$ is represented by a contour integral along the unit circle $C$, as follows:

$$
\left\langle a_{m} b_{n}\right\rangle=\frac{1}{2 \pi i} \oint_{C} \frac{2 \sqrt{r_{a}}}{\left(1+r_{a}\right) z_{p}^{k}+\left(1-r_{a}\right) z_{p}^{-k}} \frac{2 \sqrt{r_{b}}}{\left(1+r_{b}\right) z_{p}^{l}+\left(1-r_{b}\right) z_{p}^{-l}} z_{p}^{-1} d z_{p}
$$

Let $S\left(z_{p}\right)$ be an integrand of Eq. (43).

1. $r_{a}=r_{b}=1$.

$$
S\left(z_{p}\right)=z_{p}^{-k-l-1} .
$$

The order of the pole is larger than 1 . Thus, $\left\langle a_{m} b_{n}\right\rangle=0$ for all $k, l$.

2. $r_{a}=1, r_{b} \neq 1$.

$$
S\left(z_{p}\right)=\frac{\frac{2 \sqrt{r_{b}}}{1+r_{b}}}{z_{p}^{2 l}+\frac{1-r_{b}}{1+r_{b}}} z_{p}^{-k+l-1} .
$$

The following power series converges because of $\left|1-r_{b} / 1+r_{b}\right|<1$.

$$
S\left(z_{p}\right)=\frac{2 \sqrt{r_{b}}}{1+r_{b}} \sum_{q=0}^{\infty}\left(\frac{r_{b}-1}{1+r_{b}}\right)^{q} z_{p}^{-k-(2 q+1) l-1} .
$$

For all $k, l$, the residue is 0 . Thus, $\left\langle a_{m} b_{n}\right\rangle=0$ for all $k, l$.

3. $r_{a} \neq 1, r_{b}=1$.

$$
S\left(z_{p}\right)=\frac{\frac{2 \sqrt{r_{a}}}{1+r_{a}}}{z_{p}^{2 k}+\frac{1-r_{a}}{1+r_{a}}} z_{p}^{k-l-1} .
$$

The above representation is identical to Eq. (45). Thus, $\left\langle a_{m} b_{n}\right\rangle=0$ for all $k, l$. 
4. $r_{a} \neq 1, r_{b} \neq 1$.

$$
S\left(z_{p}\right)=\frac{\frac{4 \sqrt{r_{a} r_{b}}}{\left(1+r_{a}\right)\left(1+r_{b}\right)}}{\left(z_{p}^{2 k}+\frac{1-r_{a}}{1+r_{a}}\right)\left(z_{p}^{2 l}+\frac{1-r_{b}}{1+r_{b}}\right)} z_{p}^{k+l-1} .
$$

Poles are located at $z_{p}=w_{q_{a}}^{+}$or $w_{q_{a}}^{-}$, and $z_{p}=w_{q_{b}}^{+}$or $w_{q_{b}}^{-}$, such that

$$
\begin{aligned}
& w_{q_{a}}^{+}=\left(\frac{r_{a}-1}{1+r_{a}}\right)^{1 / 2 k} e^{\pi i q_{a} / k} \text { for } r_{a}>1, \\
& w_{q_{a}}^{-}=\left(\frac{1-r_{a}}{1+r_{a}}\right)^{1 / 2 k} e^{\pi i\left(2 q_{a}-1\right) / 2 k} \text { for } r_{a}<1 \\
& w_{q_{b}}^{+}=\left(\frac{r_{b}-1}{1+r_{b}}\right)^{1 / 2 l} e^{\pi i q_{b} / l} \text { for } r_{b}>1, \\
& w_{q_{b}}^{-}=\left(\frac{1-r_{b}}{1+r_{b}}\right)^{1 / 2 l} e^{\pi i\left(2 q_{b}-1\right) / 2 l} \text { for } r_{b}<1
\end{aligned}
$$

where $q_{a} \in\{1, \cdots, 2 k\}, q_{b} \in\{1, \cdots, 2 l\} .{ }^{\forall} q_{a} \in\{1, \cdots, k\} ; w_{q_{a}+k}^{ \pm}=-w_{q_{a}}^{ \pm} .{ }^{\forall} q_{b} \in\{1, \cdots, l\} ;$ $w_{q_{b}+l}^{ \pm}=-w_{q_{b}}^{ \pm}$.

(a) All poles are of order 1 .

The residues corresponding to $w_{q_{a}}^{ \pm}$and $w_{q_{b}}^{ \pm}$are

$$
\begin{aligned}
\operatorname{Res}_{z_{p}=w_{q_{a}}^{ \pm}}\left[S\left(z_{p}\right)\right] & =-\frac{2 \sqrt{r_{a} r_{b}}}{k\left(1-r_{a}\right)\left(1+r_{b}\right)} \frac{w_{q_{a}}^{ \pm}}{w_{q_{a}}^{ \pm 2 l}+\frac{1-r_{b}}{1+r_{b}}} \\
\operatorname{Res}_{z_{p}=w_{q_{b}}^{ \pm}}\left[S\left(z_{p}\right)\right] & =-\frac{2 \sqrt{r_{a} r_{b}}}{l\left(1+r_{a}\right)\left(1-r_{b}\right)} \frac{w_{q_{b}}^{ \pm k+l}}{w_{q_{b}}^{ \pm 2 k}+\frac{1-r_{a}}{1+r_{a}}} .
\end{aligned}
$$

For $k+l=$ odd, the sum of the residues corresponding to $w_{q_{a}}^{ \pm}$and $w_{q_{a}+k}^{ \pm}$vanishes, and the sum of the residues corresponding to $w_{q_{b}}^{ \pm}$and $w_{q_{b}+l}^{ \pm}$also vanishes. Thus, $\left\langle a_{m} b_{n}\right\rangle=0$ for $k+l=$ odd, and

$$
\begin{aligned}
\left\langle a_{m} b_{n}\right\rangle= & -\frac{4 \sqrt{r_{a} r_{b}}}{k\left(1-r_{a}\right)} \sum_{q_{a}=1}^{k} \frac{w_{q_{a}}^{ \pm}}{\left(1+r_{b}\right) w_{q_{a}}^{ \pm 2 l}+1-r_{b}} \\
& -\frac{4 \sqrt{r_{a} r_{b}}}{l\left(1-r_{b}\right)} \sum_{q_{b}=1}^{l} \frac{w_{q_{b}}^{ \pm}}{\left(1+r_{a}\right) w_{q_{b}}^{ \pm 2 k}+1-r_{a}}
\end{aligned}
$$

for $k+l=$ even.

(b) Poles of order 2 exist.

Poles of order 2 exist in case $\left(1-r_{a} / 1+r_{a}\right)^{1 / 2 k}=\left(1-r_{b} / 1+r_{b}\right)^{1 / 2 l}$ for $r_{a}, r_{b}<1$, or in case $\left(r_{a}-1 / 1+r_{a}\right)^{1 / 2 k}=\left(r_{b}-1 / 1+r_{b}\right)^{1 / 2 l}$ for $r_{a}, r_{b}>1$.

i. $r_{a}, r_{b}<1$

Poles of order 2 exist only for $k+l=$ odd, and are located at $z_{p}= \pm w^{-}$, such that

$$
w^{-}=i\left(\frac{1-r_{a}}{1+r_{a}}\right)^{1 / 2 k}=i\left(\frac{1-r_{b}}{1+r_{b}}\right)^{1 / 2 l} .
$$

Let $Q_{-}(z)$ be a polynomial function defined by

$$
Q_{-}(z)=\prod_{q_{a}=1, q_{a} \neq(k+1) / 2}^{k}\left(z^{2}-{w_{q_{a}}^{-}}^{2}\right) \prod_{q_{b}=1, q_{b} \neq(l+1) / 2}^{l}\left(z^{2}-{w_{q_{b}}^{-2}}^{2}\right),
$$


and the residues are represented, as follows:

$$
\begin{aligned}
\operatorname{Res}_{z_{p}=w^{-}}\left[S\left(z_{p}\right)\right] & =\frac{4 \sqrt{r_{a} r_{b}} w^{-k+l-2}}{\left(1+r_{a}\right)\left(1+r_{b}\right)} \frac{(k+l-1) Q_{-}\left(w^{-}\right)-w^{-} Q_{-}^{\prime}\left(w^{-}\right)}{Q_{-}^{2}\left(w^{-}\right)} \\
\operatorname{Res}_{z_{p}=-w^{-}}\left[S\left(z_{p}\right)\right] & =\frac{4 \sqrt{r_{a} r_{b}}\left(-w^{-}\right)^{k+l-2}}{\left(1+r_{a}\right)\left(1+r_{b}\right)} \frac{(k+l-1) Q_{-}\left(w^{-}\right)-w^{-} Q_{-}^{\prime}\left(w^{-}\right)}{Q_{-}^{2}\left(w^{-}\right)} .
\end{aligned}
$$

The sum of the two residues vanishes because $k+l$ is odd.

ii. $r_{a}, r_{b}>1$

Poles of order 2 are located at $z_{p}= \pm w^{+}$, such that

$$
w^{+}=\left(\frac{r_{a}-1}{1+r_{a}}\right)^{1 / 2 k}=\left(\frac{r_{b}-1}{1+r_{b}}\right)^{1 / 2 l} .
$$

Let $Q_{+}(z)$ be a polynomial function defined by

$$
Q_{+}(z)=\prod_{q_{a}=1}^{k-1}\left(z^{2}-w_{q_{a}}^{+2}\right) \prod_{q_{b}=1}^{l-1}\left(z^{2}-{w_{q_{b}}^{+}}^{2}\right),
$$

and the residues are represented, as follows:

$$
\begin{aligned}
\operatorname{Res}_{z_{p}=w^{+}}\left[S\left(z_{p}\right)\right] & =\frac{4 \sqrt{r_{a} r_{b}} w^{+k+l-2}}{\left(1+r_{a}\right)\left(1+r_{b}\right)} \frac{(k+l-1) Q_{+}\left(w^{+}\right)-w^{+} Q_{+}^{\prime}\left(w^{+}\right)}{Q_{+}^{2}\left(w^{+}\right)} \\
\operatorname{Res}_{z_{p}=-w^{+}}\left[S\left(z_{p}\right)\right] & =\frac{4 \sqrt{r_{a} r_{b}}\left(-w^{+}\right)^{k+l-2}}{\left(1+r_{a}\right)\left(1+r_{b}\right)} \frac{(k+l-1) Q_{+}\left(w^{+}\right)-w^{+} Q_{+}^{\prime}\left(w^{+}\right)}{Q_{+}^{2}\left(w^{+}\right)} .
\end{aligned}
$$

For $k+l=$ odd, the sum of the two residues vanishes.

The other poles give the residues represented by Eqs. (53)-(54). Thus, $\left\langle a_{m} b_{n}\right\rangle=0$ for $k+l=$ odd.

From the results in all the cases $1-4,\left\langle a_{m} b_{n}\right\rangle=0$ for $k+l=$ odd.

Theorem 5

$p_{1} \neq p_{2},{ }^{\forall} p_{1}, p_{2} \in \mathbf{N}+0 ; X\left(\boldsymbol{a}_{p_{1}}\right) \perp X\left(\boldsymbol{a}_{p_{2}}\right)$.

Proof

Assume $p_{1}>p_{2}$ without loss of generality. For $p_{2}=0,{ }^{\forall} n \in \mathbf{N} ;\left\langle a_{n}\right\rangle=0$ (theorem 2). Thus, $X\left(\boldsymbol{a}_{p_{1}}\right) \perp X\left(\boldsymbol{a}_{0}\right)$. For $p_{2}>0, X\left(\boldsymbol{a}_{p_{1}}\right)$ is spanned by $\left\{a_{M\left(p_{1}, q_{1}\right)}, a_{M\left(p_{1}, q_{1}\right)}^{*} \mid q_{1} \in \mathbf{N}\right\}$, and $X\left(\boldsymbol{a}_{p_{2}}\right)$ by $\left\{a_{M\left(p_{2}, q_{2}\right)}, a_{M\left(p_{2}, q_{2}\right)}^{*} \mid q_{2} \in \mathbf{N}\right\}$. A coprime set of $M\left(p_{1}, q_{1}\right)$ and $M\left(p_{2}, q_{2}\right)$ is $2^{p_{1}-p_{2}}\left(2 \tilde{q}_{1}-1\right)$ and $2 \tilde{q}_{2}-1$, where $2 \tilde{q}_{1}-1$ and $2 \tilde{q}_{2}-1$ are a coprime set of $2 q_{1}-1$ and $2 q_{2}-1$. The sum is odd for any pairs of $p_{1}, p_{2}$, $q_{1}$, and $q_{2}$, because $2^{p_{1}-p_{2}}$ is always even. Then, every inner product between the members in $\boldsymbol{a}_{p_{1}}$ and $\boldsymbol{a}_{p_{2}}$ is orthogonal (theorems 3 and 4). Thus, $X\left(\boldsymbol{a}_{p_{1}}\right) \perp X\left(\boldsymbol{a}_{p_{2}}\right)$.

Theorem 6

$r_{2 n-1}=r\left({ }^{\forall} n \in \mathbf{N}\right)$. Then, the following series converges absolutely.

$$
\sum_{n=1}^{\infty}\left|c_{2 n-1}\right|
$$

Proof

Let $\left\{q_{1}, \ldots, q_{N}\right\}$ be prime factors of $2 n-1$, such that $2 n-1=\prod_{l=1}^{N} q_{l}$, and $q_{1} \leq q_{2} \leq \cdots \leq q_{N}$. Let $S_{2 n-1}$ be the set of all combinations of the product of any selection from $\left\{q_{1}, \ldots, q_{N}\right\}$. Duplication of members in $S_{2 n-1}$ is not allowed. $\left\langle e_{2 m-1}^{*} a_{2 n-1}\right\rangle$ is nonzero for ${ }^{\exists} s \in \mathbf{N}$, such that $(2 s-1)(2 m-1)=$ 
$2 n-1$ (2. in proof of theorem 3). Thus, ${ }^{\forall} n \geq 2$;

$$
c_{2 n-1}=-\frac{1+r}{2 \sqrt{r}}\left(\frac{r-1}{1+r}\right)^{n-1}-\sum_{q \in S_{2 n-1} \backslash\{2 n-1\}} c_{q}\left(\frac{r-1}{1+r}\right)^{\frac{1}{2}\left(\frac{2 n-1}{q}-1\right)} .
$$

If $2 n-1$ is a prime number, $S_{2 n-1}=\{2 n-1\}$. Then, $c_{2 n-1}=-\frac{1+r}{2 \sqrt{r}}\left(\frac{r-1}{1+r}\right)^{n-1}$. For the other cases, ${ }^{\exists} C_{2 n-1} \in(0, \infty)$, such that

$$
\left|c_{2 n-1}\right|<C_{2 n-1}\left|c_{2 n-1 / q_{1}}\right|\left|\frac{r-1}{1+r}\right|^{\frac{1}{2}\left(q_{1}-1\right)} .
$$

The relation is recursively applied to $\left|c_{2 n-1 / q_{1}}\right|,\left|c_{2 n-1 / q_{1} q_{2}}\right|$, etc. Thus, $\left|c_{2 n-1}\right|$ satisfies the following condition, ${ }^{\exists} C \in(0, \infty)$, such that

$$
\left|c_{2 n-1}\right|<C\left|\frac{r-1}{1+r}\right|^{\frac{1}{2}\left(\sum_{l=1}^{N} q_{l}-N\right)} .
$$

From the theorem of prime factorization, the upper limit of $N$ is $\log (2 n-1) / \log 3$, and the lower limit of $\sum q_{l}$ is $3 \log (2 n-1) / \log 3$. Therefore, ${ }^{\forall} n \in \mathbf{N} ;{ }^{\exists} C \in(0, \infty)$, such that

$$
\left|c_{2 n-1}\right|<C\left|\frac{r-1}{1+r}\right|^{\log (2 n-1) / \log 3} .
$$

Thus, d'Alembert's ratio test $\left(\left|c_{2 n+1} / c_{2 n-1}\right|<1\right)$ indicates that $\sum_{n=1}^{\infty}\left|c_{2 n-1}\right|$ converges absolutely.

Theorem 7

$r_{2 n-1}=r\left({ }^{\forall} n \in \mathbf{N}\right)$. Then, the following series uniformly converges

$$
\sum_{n=1}^{\infty} c_{2 n-1} a_{2 n-1}
$$

Proof

${ }_{n \in \mathbf{N}} ;\left|a_{2 n-1}\right| \leq \max (\sqrt{r}, 1 / \sqrt{r})<\infty$. Then, ${ }^{\exists} A \in(0, \infty)$, such that

$$
\sum_{n=1}^{\infty}\left|c_{2 n-1} a_{2 n-1}\right| \leq A \sum_{n=1}^{\infty}\left|c_{2 n-1}\right| \text {. }
$$

From theorem $6, \sum_{n=1}^{\infty}\left|c_{2 n-1}\right|$ converges absolutely. Thus, the series (69) uniformly converges.

Theorem 8

$r_{2 n-1}=r\left({ }^{\forall} \in \mathbf{N}\right) \Rightarrow$

$$
e_{1}=\sum_{n=1}^{\infty} c_{2 n-1} a_{2 n-1}
$$

Proof

Let $d_{2 n-1}$ be a complex coefficient. Assume $e_{1}=\sum_{n=1}^{\infty} d_{2 n-1} a_{2 n-1}$, and also assume its uniform convergence. $\left\langle e_{1} e_{1}^{*}\right\rangle=d_{1}\left\langle a_{1} e_{1}^{*}\right\rangle=1$, and

$$
\left\langle e_{1} e_{2 n-1}^{*}\right\rangle=\sum_{m=1}^{n} d_{2 m-1}\left\langle a_{2 m-1} e_{2 n-1}^{*}\right\rangle=0
$$

for $n \geq 2$. Then, $d_{n}=c_{n}(n \in \mathbf{N})$. The series uniformly converges as proved in theorem 7 .

Theorem 9

$r_{n}=r\left({ }^{\forall} n \in \mathbf{N}\right) \Rightarrow$

$$
e_{n}=\sum_{m=1}^{\infty} c_{2 m-1} a_{(2 m-1) n} \quad\left({ }^{\forall} n \in \mathbf{N}\right)
$$


Proof

Let $e_{1}(\omega)$ and $a_{2 m-1}(\omega)$ be defined in $\omega \in[0, n \Omega](n \in \mathbf{N})$, and $\omega_{n}=\omega / n$. From the definition, $e_{1}(\omega)$ for $\omega \in[0, \Omega]$ is equal to $e_{1}(n \Omega+\omega)(n \in \mathbf{N})$, and $a_{2 m-1}(\omega)=a_{2 m-1}(n \Omega+\omega)$. This indicates that the series expansion of $e_{1}$ (theorem 8) can be defined in $\omega \in[0, n \Omega] . e_{1}\left(n \omega_{n}\right)$ and $a_{2 m-1}\left(n \omega_{n}\right)$ are equal to $e_{n}\left(\omega_{n}\right)$ and $a_{(2 m-1) n}\left(\omega_{n}\right)$, respectively. Thus, the series expansion by Eq. (73) is available for $\omega \in[0, \Omega]$.

\section{Theorem 10}

$r_{n}=r\left({ }^{\forall} \in \mathbf{N}\right) \Rightarrow \bigoplus_{p=0}^{\infty} X\left(\boldsymbol{a}_{p}\right)=L^{2}$.

Proof

${ }^{\forall} p, q \in \mathbf{N} ; e_{M(p, q)}=\sum_{m=1}^{\infty} c_{2 m-1} a_{(2 m-1) M(p, q)}$ (theorem 9). ${ }^{\forall} m, q \in \mathbf{N} ;{ }^{\exists} q_{m} \in \mathbf{N}$, such that $2 q_{m}-$ $1=(2 q-1)(2 m-1)$. Then, $a_{(2 m-1) M(p, q)}$ is a basis belonging to $X\left(\boldsymbol{a}_{p}\right)$ because of $M\left(p, q_{m}\right)=(2 m-$ 1) $M(p, q)$. Thus, all the bases $e_{M(p, q)}$ in $X_{p}^{F}$ can be represented by a series of the bases in $X\left(\boldsymbol{a}_{p}\right)$. This indicates $X\left(\boldsymbol{a}_{p}\right) \supseteq X_{p}^{F}$. Therefore, $X\left(\boldsymbol{a}_{p}\right)=X_{p}^{F}$ for all $p \in \mathbf{N}$. Thus, $\bigoplus_{p=0}^{\infty} X\left(\boldsymbol{a}_{p}\right)=L^{2}$. 